\title{
NORMALIZERS OF OPERATOR ALGEBRAS AND REFLEXIVITY
}

\author{
A. KATAVOLOS AND I.G. TODOROV
}

\begin{abstract}
The set of normalizers between von Neumann (or, more generally, reflexive) algebras $\mathcal{A}$ and $\mathcal{B}$, (that is, the set of all operators $T$ such that $T \mathcal{A} T^{*} \subseteq \mathcal{B}$ and $T^{*} \mathcal{B} T \subseteq \mathcal{A}$ ) possesses 'local linear structure': it is a union of reflexive linear spaces. These spaces belong to the interesting class of normalizing linear spaces, namely, those linear spaces $\mathcal{U}$ satisfying $\mathcal{U U}^{*} \mathcal{U} \subseteq \mathcal{U}$. Such a space is reflexive whenever it is ultraweakly closed, and then it is of the form $\mathcal{U}=\{T: T L=\phi(L) T$ for all $L \in \mathcal{L}\}$ where $\mathcal{L}$ is a set of projections and $\phi$ a certain map defined on $\mathcal{L}$. A normalizing space consists of normalizers between appropriate von Neumann algebras $\mathcal{A}$ and $\mathcal{B}$. Necessary and sufficient conditions are found for a normalizing space to consist of normalizers between two reflexive algebras. Normalizing spaces which are bimodules over maximal abelian selfadjoint algebras consist of operators 'supported' on sets of the form $[f=g]$ where $f$ and $g$ are appropriate Borel functions. They also satisfy spectral synthesis in the sense of Arveson.
\end{abstract}

\section{INTRODUCTION AND PRELIMINARIES}

This paper is devoted to the study of a class of linear spaces of operators on Hilbert space which arise as normalizers of operator algebras; we call them normalizing subspaces.

Normalizers of von Neumann algebras (in particular, of maximal abelian selfadjoint algebras - masas for short) are known to play an important role in various contexts. The non-selfadjoint generalizations of von Neumann algebras are the reflexive algebras first introduced by Halmos in 88. We will primarily be concerned with normalizers of such algebras. Recall that an operator $T$ normalizes a reflexive (not necessarily selfadjoint) algebra $\mathcal{A}$ if $T$ and its adjoint satisfy $T^{*} \mathcal{A} T \subseteq \mathcal{A}$. The set of all normalizers of $\mathcal{A}$ is of course not a linear space in general. However, it turns out that the action of a normalizer $T$ and its adjoint $T^{*}$ on the invariant projections of $\mathcal{A}$ defines a linear space of operators all of which normalize $\mathcal{A}$. Moreover, this space is reflexive in the terminology of Loginov-Shulman [12] and Erdos [6] and is closed under the 'triple product' $A B^{*} C$. Thus the set of normalizers of a reflexive (not necessarily selfadjoint) algebra (and indeed, the set of semi-normalizers between two reflexive algebras - see section 5) appears as the union of reflexive linear spaces which have additional algebraic structure. It is the interplay between linearity and normalization that forms the subject matter of the present work. For example, we show that every normalizer is the norm-limit of linear combinations of normalizing partial isometries, and every compact normalizer is the limit of finite rank normalizers. We also show that the sum of two normalizers of CSL algebras is again a normalizer only

1991 Mathematics Subject Classification. Primary 47L05; Secondary 47L35, 46L10.

Key words and phrases. Operator algebras, reflexivity, normalizers, masa-bimodules.

The second-named author was supported by a grant from the Greek National Scholarship Foundation. 
when both are contained in a single reflexive masa bimodule consisting of normalizers, and obtain generalizations of the results of Coates [3] on normalizers of nest algebras.

These observations lead us to introduce the class of normalizing spaces, namely, those linear spaces of operators which are closed under the 'triple product' $A B^{*} C$. These subspaces are interesting in their own right; they generalize selfadjoint algebras of operators, and share many properties in common with such algebras. For instance, they satisfy an analogue of the bicommutant theorem: they are reflexive whenever they are ultraweakly closed (unlike general non-selfadjoint algebras). Alternatively given a set $\mathcal{L}$ of projections and a map $\phi$ defined on $\mathcal{L}$, the set of all operators $T$ satisfying $T L=\phi(L) T$ for all $L \in \mathcal{L}$ is a normalizing subspace, and every ultraweakly closed normalizing subspace is of this form.

A normalizing subspace $\mathcal{U}$ is a bimodule over the selfadjoint algebras $\left[\mathcal{U}^{*} \mathcal{U}\right]$ and $\left[\mathcal{U U}^{*}\right]$ and induces a complete lattice isomorphism $\chi$ between the invariant projections of the 'non-degenerate parts' of these algebras. Also, $\mathcal{U}$ normalizes the first algebra into the second (that is, $T\left(\mathcal{U}^{*} \mathcal{U}\right) T^{*} \subseteq \mathcal{U} \mathcal{U}^{*}$ and $T^{*}\left(\mathcal{U} \mathcal{U}^{*}\right) T \subseteq \mathcal{U}^{*} \mathcal{U}$ for each $T \in \mathcal{U}$ ). Conversely, we are able to characterize when $\mathcal{U}$ normalizes a pair of reflexive (not necessarily selfadjoint) algebras $\mathcal{A}$ and $\mathcal{B}$. Apart from the obvious relations $\mathcal{U}^{*} \mathcal{U} \subseteq \mathcal{A}$ and $\mathcal{U U}^{*} \subseteq \mathcal{B}$, the map $\chi$ must induce a bijection between the invariant projection lattices of the 'non-degenerate parts' of these algebras.

Thus, if $\mathcal{U}$ is a normalizing space then the non-degenerate parts of the von Neumann algebras generated by $\left[\mathcal{U}^{*} \mathcal{U}\right]$ and $\left[\mathcal{U U}^{*}\right]$ are Morita equivalent in the sense of Rieffel [14. Conversely, if $\mathcal{A}$ and $\mathcal{B}$ are Morita equivalent $\mathrm{W}^{*}$-algebras, then there are faithful representations of $\mathcal{A}$ and $\mathcal{B}$ such that the bimodule which establishes the equivalence is represented as a normalizing space $\mathcal{U}$ of operators between the respective Hilbert spaces. In this paper our concern is not with the notion of Morita equivalence of (abstract) $\mathrm{W}^{*}$-algebras, but rather with the properties of normalizers between (concrete) reflexive algebras and especially with the interplay between normalizers and reflexivity. Notice, however, that this connection between normalizers and Morita equivalence might not have been observed had we considered normalizers of a single algebra.

We prove that normalizing subspaces which are bimodules over two maximal abelian selfadjoint algebras consists of operators 'supported' on sets of the form $[f=g]$ where $f$ and $g$ are appropriate Borel functions. This includes the case of normalizing subspaces which are generated by rank one operators. In case one of the algebras $\left[\mathcal{U}^{*} \mathcal{U}\right],\left[\mathcal{U U}^{*}\right]$ is abelian, the support of $\mathcal{U}$ turns out to be the 'graph' or the 'reverse graph' of a Borel function. We also show that normalizing masa-bimodules satisfy spectral synthesis in the sense of Arveson [1]. This gives a clear geometric description of the normalizers of a CSL algebra in terms of generalized graphs or partial graphs. These partial graphs are analogous to the ones appearing in the work of Feldman and Moore [7] and others. In these papers, only partial isometries normalizing certain Cartan masas are considered, while in our work the emphasis is on the whole reflexive linear space generated by each generalised graph. Also, we deal with arbitrary (nonabelian and non-selfadjoint) CSL algebras.

The notation we use is standard; see for example [4]. We review some definitions and facts from [6] and [12]. Let $\mathcal{H}_{1}$ and $\mathcal{H}_{2}$ be complex Hilbert spaces, $\mathcal{P}_{i}$ the lattice of all (orthogonal) projections on $\mathcal{H}_{i}, i=1,2$. We let $\mathcal{M}\left(\mathcal{P}_{1}, \mathcal{P}_{2}\right)$ denote the set of all 
maps $\varphi: \mathcal{P}_{1} \rightarrow \mathcal{P}_{2}$ which are 0 -preserving and $\vee$-continuous (i.e. preserve arbitrary suprema). Erdos [6] shows that each $\varphi \in \mathcal{M}\left(\mathcal{P}_{1}, \mathcal{P}_{2}\right)$ uniquely defines semi-lattices $\mathcal{S}_{1 \varphi} \subseteq \mathcal{P}_{1}$ and $\mathcal{S}_{2 \varphi}=\varphi\left(\mathcal{P}_{1}\right) \subseteq \mathcal{P}_{2}$ such that $\varphi$ is a bijection between $\mathcal{S}_{1 \varphi}$ and $\mathcal{S}_{2 \varphi}$ and is uniquely determined by its restriction to $\mathcal{S}_{1 \varphi}$. Moreover, $\mathcal{S}_{1 \varphi}$ is meet-complete and contains the identity projection while $\mathcal{S}_{2 \varphi}$ is join-complete and contains the zero projection.

Note that the set

$$
\text { Op } \varphi=\left\{T \in \mathcal{B}\left(\mathcal{H}_{1}, \mathcal{H}_{2}\right): \varphi(P)^{\perp} T(P)=0 \text { for each } P \in \mathcal{P}_{1}\right\}
$$

is also uniquely determined by $\left.\varphi\right|_{\mathcal{S}_{1 \varphi}}$ : if $T$ satisfies $\varphi(P)^{\perp} T(P)=0$ for each $P \in \mathcal{S}_{1 \varphi}$, then $T \in \operatorname{Op} \varphi$.

Given a subspace $\mathcal{U} \subseteq \mathcal{B}\left(\mathcal{H}_{1}, \mathcal{H}_{2}\right)$, we define its map Map $\mathcal{U}: \mathcal{P}_{1} \rightarrow \mathcal{P}_{2}$ by

$$
(\operatorname{Map} \mathcal{U})(P)=\overline{[\mathcal{U}(P)]} \quad\left(P \in \mathcal{P}_{1}\right)
$$

(where, here and in the sequel, the symbol $\overline{[\mathcal{U}(P)]}$ will stand for the projection onto the closed subspace spanned by $\left.\left\{S x: x \in P\left(\mathcal{H}_{1}\right), S \in \mathcal{U}\right\}\right)$.

If $\varphi^{*}=\operatorname{Map} \mathcal{U}^{*}$, then $\mathcal{S}_{1 \varphi}=\left\{P^{\perp}: P \in \varphi^{*}\left(\mathcal{P}_{2}\right)\right\}[6]$.

The reflexive hull $\operatorname{Ref} \mathcal{U}$ of $\mathcal{U}$ is defined to be the space

$$
\operatorname{Ref} \mathcal{U}=\left\{T \in \mathcal{B}\left(\mathcal{H}_{1}, \mathcal{H}_{2}\right): T x \in \overline{\mathcal{U}} x \text {, for each } x \in \mathcal{H}_{1}\right\}
$$

[12]. A subspace $\mathcal{U}$ is called reflexive if $\mathcal{U}=\operatorname{Ref} \mathcal{U}$. It is easily seen that $\operatorname{Ref} \mathcal{U}=$ Op Map $\mathcal{U}$ [6]. A unital algebra $\mathcal{A} \subseteq \mathcal{B}(\mathcal{H})$ is reflexive if and only if it is of the form $\mathcal{A}=\operatorname{Alg} \mathcal{L}=\left\{A: L^{\perp} A L=0\right.$ for all $\left.L \in \mathcal{L}\right\}$, where $\mathcal{L}=$ Lat $\mathcal{A}$ is the complete lattice of all invariant projections of $\mathcal{A}$.

Given $\varphi \in \mathcal{M}\left(\mathcal{P}_{1}, \mathcal{P}_{2}\right)$ the subspace

$$
\mathcal{V}=\left\{T \in \mathcal{B}\left(\mathcal{H}_{1}, \mathcal{H}_{2}\right): \varphi(L) T\left(L^{\perp}\right)=0 \text { for each } L \in \mathcal{S}_{1 \varphi}\right\}
$$

is clearly reflexive. We denote its map by $\varphi^{\perp}$. Thus $\mathcal{V}=\operatorname{Op} \varphi^{\perp}$ and $\varphi^{\perp} \in \mathcal{M}\left(\mathcal{P}_{1}, \mathcal{P}_{2}\right)$ satisfies $\varphi^{\perp}\left(L^{\perp}\right) \leq \varphi(L)^{\perp}$ for each $L \in \mathcal{S}_{1 \varphi}$.

The following simple observations, whose proofs are routine, will be used repeatedly.

Lemma 1.1. Let $\mathcal{U} \subseteq \mathcal{B}\left(\mathcal{H}_{1}, \mathcal{H}_{2}\right)$ be a subspace and $\mathcal{A}_{i} \subseteq \mathcal{B}\left(\mathcal{H}_{i}\right)(i=1,2)$ be unital algebras such that $\mathcal{A}_{2} \mathcal{U} \mathcal{A}_{1} \subseteq \mathcal{U}$. If $\varphi=\operatorname{Map} \mathcal{U}$, then $\mathcal{S}_{i \varphi} \subseteq$ Lat $\mathcal{A}_{i}$. Thus $\mathcal{A}_{1} \subseteq$ $\operatorname{Alg} \mathcal{S}_{1 \varphi}$ and $\mathcal{A}_{2} \subseteq \operatorname{Alg} \mathcal{S}_{2 \varphi}$. If additionally $\mathcal{U}$ is reflexive, then the algebra $\operatorname{Alg} \mathcal{S}_{1 \varphi}$ (resp. $\left.\operatorname{Alg} \mathcal{S}_{2 \varphi}\right)$ is the largest algebra over which $\mathcal{U}$ is a right (resp. left) module.

\section{Normalizing SPACES OF OPERATORS}

The notion of reflexivity for subspaces generalizes the corresponding notion defined by Halmos [8] for unital algebras. Among reflexive algebras, the selfadjoint ones, namely the von Neumann algebras, have of course a distinguished place. Note that a unital algebra $\mathcal{A}$ is selfadjoint if and only if $\mathcal{A} \mathcal{A}^{*} \mathcal{A} \subseteq \mathcal{A}$. As the results of this paper show, the generalization of this property to subspaces is particularly fruitful.

Definition 2.1. A subspace $\mathcal{U} \subseteq \mathcal{B}\left(\mathcal{H}_{1}, \mathcal{H}_{2}\right)$ is said to be normalizing if it is closed under the 'triple product' $(A, B, C) \rightarrow A B^{*} C$. 
Remark 2.2. For a subspace $\mathcal{U} \subseteq \mathcal{B}\left(\mathcal{H}_{1}, \mathcal{H}_{2}\right)$, the following are equivalent:

(i) $\mathcal{U}$ is normalizing.

(ii) There is a unital ${ }^{*}$-algebra $\mathcal{A}_{1} \subseteq \mathcal{B}\left(\mathcal{H}_{1}\right)$ such that $\mathcal{U} \mathcal{A}_{1} \subseteq \mathcal{U}$ and $\mathcal{U}^{*} \mathcal{U} \subseteq \mathcal{A}_{1}$.

(iii) There is a subspace $\mathcal{A}_{1} \subseteq \mathcal{B}\left(\mathcal{H}_{1}\right)$ such that $\mathcal{U} \mathcal{A}_{1} \subseteq \mathcal{U}$ and $\mathcal{U}^{*} \mathcal{U} \subseteq \mathcal{A}_{1}$.

(iv) There is a unital ${ }^{*}$-algebra $\mathcal{A}_{2} \subseteq \mathcal{B}\left(\mathcal{H}_{2}\right)$ such that $\mathcal{A}_{2} \mathcal{U} \subseteq \mathcal{U}$ and $\mathcal{U U}^{*} \subseteq \mathcal{A}_{2}$.

(v) There is a subspace $\mathcal{A}_{2} \subseteq \mathcal{B}\left(\mathcal{H}_{2}\right)$ such that $\mathcal{A}_{2} \mathcal{U} \subseteq \mathcal{U}$ and $\mathcal{U U}^{*} \subseteq \mathcal{A}_{2}$.

Proof. (i) $\Rightarrow$ (ii) Let $\mathcal{A}_{1} \subseteq \mathcal{B}\left(\mathcal{H}_{1}\right)$ be the linear span of $\mathcal{U}_{1}=\left\{S^{*} T: S, T \in \mathcal{U}\right\} \cup\{I\}$. Since $\mathcal{U} \mathcal{U}^{*} \mathcal{U} \subseteq \mathcal{U}$, one verifies that $\mathcal{U}_{1}$ is a unital ${ }^{*}$-semigroup and so $\mathcal{A}_{1}$ is a unital ${ }^{*}$-subalgebra. The properties $\mathcal{U}^{*} \mathcal{U} \subseteq \mathcal{A}_{1}$ and $\mathcal{U} \mathcal{A}_{1} \subseteq \mathcal{U}$ are immediate.

(ii) $\Rightarrow$ (iii) is trivial.

(iii) $\Rightarrow$ (i) If $S, T, R \in \mathcal{U}$ then $T^{*} R \in \mathcal{A}_{1}$ and hence $S\left(T^{*} R\right) \in \mathcal{U}$.

The implications (i) $\Rightarrow$ (iv) $\Rightarrow(\mathrm{v}) \Rightarrow(\mathrm{i})$ are equally easy. $\diamond$

Remark Let $\mathcal{U}$ be a normalizing subspace, and write $\mathcal{A}$ and $\mathcal{B}$ for the algebras generated by $\mathcal{U}^{*} \mathcal{U}$ and $\mathcal{U} \mathcal{U}^{*}$ respectively. Then $\mathcal{U}$ normalizes $\mathcal{B}$ into $\mathcal{A}$, in the sense that $T^{*} \mathcal{B} T \subseteq \mathcal{A}$ and $T \mathcal{A} T^{*} \subseteq \mathcal{B}$ for all $T \in \mathcal{U}$. Conversely, if $\mathcal{U}$ is a subspace of operators, which normalizes an algebra into another, then, as we shall prove (see Proposition 5.10, $\mathcal{U}$ is contained in a normalizing space of operators.

If $\mathcal{A}$ is a unital selfadjoint algebra, then its invariant subspace lattice $\mathcal{L}$ is orthocomplemented; thus its map $\chi=\operatorname{Map}(\mathcal{A})$ (namely, the identity map of $\mathcal{L}$ ) preserves orthogonality. This property characterizes maps of normalizing subspaces.

Definition 2.3. A map $\chi \in \mathcal{M}\left(\mathcal{P}_{1}, \mathcal{P}_{2}\right)$ is said to be an ortho-map if $\chi(L) \perp \chi\left(L^{\perp}\right)$ for each $L \in \mathcal{S}_{1 \chi}$.

The following theorem shows the connection between ortho-maps and normalizing spaces. Statement (b)(ii) corresponds to the von Neumann Bicommutant Theorem.

Theorem 2.4. (a) Let $\varphi \in \mathcal{M}\left(\mathcal{P}_{1}, \mathcal{P}_{2}\right)$ and

$$
\mathcal{U}=\left\{T \in \mathcal{B}\left(\mathcal{H}_{1}, \mathcal{H}_{2}\right): T L=\varphi(L) T \text { for each } L \in \mathcal{S}_{1 \varphi}\right\} .
$$

Then $\mathcal{U}=\operatorname{Op} \varphi \cap \mathrm{Op} \varphi^{\perp}$ is a normalizing space.

(b) Let $\mathcal{U} \subseteq \mathcal{B}\left(\mathcal{H}_{1}, \mathcal{H}_{2}\right)$ be a normalizing subspace. Then

(i) $\mathrm{Map} \mathcal{U}$ is an ortho-map and

(ii) $\operatorname{Ref}(\mathcal{U})=c l_{W O T}(\mathcal{U})=c l_{\text {uw }}(\mathcal{U})$.

(c) Let $\chi \in \mathcal{M}\left(\mathcal{P}_{1}, \mathcal{P}_{2}\right)$ be an ortho-map. Then

$$
\text { Op } \chi=\left\{T \in \mathcal{B}\left(\mathcal{H}_{1}, \mathcal{H}_{2}\right): T L=\chi(L) T \text { for each } L \in \mathcal{S}_{1 \chi}\right\} .
$$

Proof. (a) The equality $\mathcal{U}=\mathrm{Op} \varphi \cap \mathrm{Op} \varphi^{\perp}$ is easily verified.

Let $S, T, R \in \mathcal{U}$. Then for each $L \in \mathcal{S}_{1 \varphi}$ we have

$$
\left(S T^{*} R\right) L=S T^{*} \varphi(L) R=S L T^{*} R=\varphi(L)\left(S T^{*} R\right)
$$

and so $S T^{*} R \in \mathcal{U}$ which shows that $\mathcal{U}$ is normalizing.

(b) Let $\mathcal{A}_{i} \subseteq \mathcal{B}\left(\mathcal{H}_{i}\right)$ be unital *-algebras such that $\mathcal{U}^{*} \mathcal{U} \subseteq \mathcal{A}_{1}, \mathcal{U U}^{*} \subseteq \mathcal{A}_{2}$ and $\mathcal{A}_{2} \mathcal{U} \mathcal{A}_{1} \subseteq \mathcal{U}$ (Remark 2.2).

(i) Let $\chi=\operatorname{Map} \mathcal{U}$. We show that $\chi(L) \perp \chi\left(L^{\perp}\right)$ for each $L \in$ Lat $\mathcal{A}_{1}$ (this will suffice since $\mathcal{S}_{1 \chi} \subseteq$ Lat $\mathcal{A}_{1}$ by Lemma 1.1). Indeed, for each $S, T \in \mathcal{U}$ we have $S^{*} T \in \mathcal{A}_{1}$ and so, if $\xi \in L$ and $\eta \in L^{\perp}$ then

$$
\langle T \xi, S \eta\rangle=\left\langle S^{*} T \xi, \eta\right\rangle=0 .
$$


This shows that $T \xi \perp \chi\left(L^{\perp}\right)$ for each $T \in \mathcal{U}$ and hence $\chi(L) \perp \chi\left(L^{\perp}\right)$.

(ii) The properties $\mathcal{U}^{*} \mathcal{U} \subseteq \mathcal{A}_{1}, \mathcal{U U}^{*} \subseteq \mathcal{A}_{2}$ and $\mathcal{A}_{2} \mathcal{U} \mathcal{A}_{1} \subseteq \mathcal{U}$ ensure that the set

$$
\mathcal{C}=\left(\begin{array}{cc}
\mathcal{A}_{2} & \mathcal{U} \\
\mathcal{U}^{*} & \mathcal{A}_{1}
\end{array}\right)=\left\{\left(\begin{array}{cc}
B & T \\
S^{*} & A
\end{array}\right): A \in \mathcal{A}_{1}, B \in \mathcal{A}_{2}, S, T \in \mathcal{U}\right\}
$$

is a unital *-subalgebra of $\mathcal{B}\left(\mathcal{H}_{2} \oplus \mathcal{H}_{1}\right)$ and so $\operatorname{cl}_{W O T}(\mathcal{C})=\mathrm{cl}_{u w}(\mathcal{C})=\mathcal{C}^{\prime \prime}$ by the von Neumann bicommutant theorem. But, since $\mathcal{C}$ is a unital *algebra, it is easy to verify that $\mathcal{C}^{\prime \prime}=\operatorname{Alg} \operatorname{Lat} \mathcal{C}=\operatorname{Ref}(\mathcal{C})$. This implies in particular that $\operatorname{cl}_{W O T}(\mathcal{U})=$ $\operatorname{cl}_{u w}(\mathcal{U})=\operatorname{Ref}(\mathcal{U})$.

(c) If $T \in \mathcal{B}\left(\mathcal{H}_{1}, \mathcal{H}_{2}\right)$ satisfies $T L=\chi(L) T$ for each $L \in \mathcal{S}_{1 \chi}$, then $T L=\chi(L) T L$ and so $T \in \mathrm{Op} \chi$ since the latter is determined by $\mathcal{S}_{1 \chi}$. If $\chi$ is an ortho-map and $T \in \mathrm{Op} \chi$, then for each $L \in \mathcal{S}_{1 \chi}$ the relation $T L^{\perp}=\chi\left(L^{\perp}\right) T L^{\perp}$ gives $\chi(L) T L^{\perp}=$ $\chi(L) \chi\left(L^{\perp}\right) T L^{\perp}=0$ since $\chi(L) \perp \chi\left(L^{\perp}\right)$. Adding to this the relation $T L=\chi(L) T L$ gives $T L=\chi(L) T$ as required. $\diamond$

We isolate two consequences of this theorem for emphasis.

Corollary 2.5. (i) The $w^{*}$-closure of a normalizing subspace is reflexive and coincides with its WOT-closure.

(ii) A reflexive subspace is normalizing if and only if its map is an ortho-map.

Remarks We do not know whether a WOT-closed subspace whose map is an orthomap must be normalizing. Note that the map of a unital algebra is an ortho-map if and only if it its invariant subspace lattice is orthocomplemented. Thus the question, within the class of unital algebras, reduces to the well-known reductive algebra problem [13]: must a WOT-closed algebra whose invariant lattice is orthocomplemented be selfadjoint?

A subspace whose map is an ortho-map need not be normalizing. Indeed there exist nonselfadjoint transitive algebras (even triangular ones - see [10]). However, as we show below (Corollary 4.3) an ultraweakly closed subspace whose map is an ortho-map must be normalizing, provided it is a masa bimodule.

A crucial property of von Neumann algebras is that they are generated by their projections. Of course, normalizing spaces need not contain any (nontrivial) projections; their role is played by the partial isometries.

Proposition 2.6. If $\mathcal{U}$ is an ultraweakly closed normalizing space and $A=U|A|$ is the polar decomposition of an element of $\mathcal{U}$, then $U$ is a partial isometry in $\mathcal{U}$ and $U f(|A|) \in \mathcal{U}$, for every Borel function $f$ on the spectrum $\operatorname{sp}(|A|)$ of $|A|$. Moreover, $\mathcal{U}$ is the norm-closed linear span of the partial isometries it contains.

Proof. $\quad$ Let $\mathcal{A}_{1}$ be a von Neumann algebra with the property $\mathcal{U}^{*} \mathcal{U} \subseteq \mathcal{A}_{1}$ and $\mathcal{U} \mathcal{A}_{1} \subseteq \mathcal{U}$. Then $A^{*} A \in \mathcal{A}_{1}$ and so $|A| \in \mathcal{A}_{1}$. We have $U=\mathrm{w}^{*}-\lim _{\varepsilon \rightarrow 0} A(|A|+\varepsilon)^{-1}$ and so $U \in \mathcal{U}$. Since $|A| \in \mathcal{A}_{1}$, for every Borel function $f$ on $\operatorname{sp}(|A|)$, the operator $f(|A|)$ is in $\mathcal{A}_{1}$ as well and since $\mathcal{U}$ is a right $\mathcal{A}_{1}$-module, it follows that $U f(|A|) \in \mathcal{U}$.

Given $A \in \mathcal{U}$ and $\epsilon>0$, there are spectral projections $P_{1}, \ldots, P_{n}$ of $|A|$ and scalars $c_{1}, \ldots, c_{n}$ such that $\left\||A|-\sum c_{i} P_{i}\right\|<\epsilon$. Thus $\left\|A-\sum c_{i} U P_{i}\right\|<\epsilon$ and each $U P_{i}$ is a partial isometry in $\mathcal{U}$, since the initial projection of $U$ is the range projection of $|A|$. 
$\mathrm{A} \mathrm{w}^{*}$-closed normalizing subspace need not contain (nonzero) finite rank operators. We show that then it cannot contain compact operators, contrary to the situation in general reflexive subspaces [9].

Corollary 2.7. Let $\mathcal{U}$ be an ultraweakly closed normalizing space and suppose that $K$ is a compact operator in $\mathcal{U}$. Then $K$ can be approximated in the norm topology by finite rank operators in $\mathcal{U}$. Moreover, if $K$ belongs to some Schatten class $\mathcal{C}_{p}$ then it can be approximated by finite rank operators in $\mathcal{U}$ in the p-norm topology.

Proof. Immediate from Proposition 2.6. $\diamond$

Let us note that the last corollary can be inferred in a different way. Namely, consider the "matrix" algebra $\mathcal{C}$ defined in the proof of Theorem 2.4. Then $\mathcal{C}$ is a von Neumann algebra (Remark 2.2) and the result is clear for $\mathcal{C}$.

If $\mathcal{U}$ is a normalizing space, then its rank one subspace (i.e. the linear span of the rank one operators contained in $\mathcal{U}$ ) is also normalizing. The next proposition characterizes this subspace in terms of the map of $\mathcal{U}$. We denote the rank one operator sending $\xi$ to $\langle\xi, x\rangle y$ by the symbol $y \otimes x^{*}$.

Proposition 2.8. Let $\chi$ be an ortho-map. A rank one operator $y \otimes x^{*}$ belongs to $\mathrm{Op} \chi$ if and only if, for each $L \in \mathcal{S}_{1 \chi}$,

$$
L x \neq 0 \Leftrightarrow L^{\perp} x=0 \Leftrightarrow \chi(L)^{\perp} y=0 \Leftrightarrow \chi(L) y \neq 0 .
$$

Proof. By Theorem 2.4, Op $\chi=\left\{T \in \mathcal{B}\left(\mathcal{H}_{1}, \mathcal{H}_{2}\right): T L=\chi(L) T, L \in \mathcal{S}_{1 \chi}\right\}$. Suppose that the rank one operator $y \otimes x^{*}$ belongs to Op $\chi$. Then, for each $L \in \mathcal{S}_{1 \chi},\left(\chi(L)^{\perp} y\right) \otimes$ $(L x)^{*}=\chi(L)^{\perp}\left(y \otimes x^{*}\right) L=0$ and $(\chi(L) y) \otimes\left(L^{\perp} x\right)^{*}=\chi(L)\left(y \otimes x^{*}\right) L^{\perp}=0$. So for each $L \in \mathcal{S}_{1 \chi}$, (either $\chi(L)^{\perp} y=0$ or $L x=0$ ) and (either $\chi(L) y=0$ or $L^{\perp} x=0$ ). If $L x \neq 0$ and $L^{\perp} x \neq 0$, then by the above conditions, we conclude that $\chi(L)^{\perp} y=$ $\chi(L) y=0$, so $y=0$, which is impossible. Because $L x$ and $L^{\perp} x$ cannot both be zero, we conclude that $L x \neq 0 \Leftrightarrow L^{\perp} x=0$. In the same way $\chi(L)^{\perp} y \neq 0 \Leftrightarrow \chi(L) y=0$. Similarly, if $L x \neq 0$, then $\chi(L)^{\perp} y=0$ and, conversely, if $\chi(L)^{\perp} y=0$, then $\chi(L) y \neq 0$ and so $L^{\perp} x=0$. The converse is trivial. $\diamond$

We would now like to show how the general theory of Erdos [6] specializes in the case of ortho-maps.

Definition 2.9. For a map $\chi$, set $I_{-}=\chi(I) \in \mathcal{P}_{2}$ and $0_{+}=\vee\{P: \chi(P)=0\} \in \mathcal{P}_{1}$. The map $\chi$ will be called essential if $0_{+}=0$ and $I_{-}=I$. A subspace of operators $\mathcal{U}$ will be called essential, if Map $\mathcal{U}$ is essential.

Theorem 2.10. Suppose that $\mathcal{U}$ is a normalizing space of operators and let $\chi=$ Map $\mathcal{U}$. Then the semi-lattices $\mathcal{S}_{1}$ and $\mathcal{S}_{2}$ of $\chi$ are complete ortho-lattices and $\chi$ is a complete ortho-lattice isomorphism of $\mathcal{S}_{1}$ onto $\mathcal{S}_{2}$. Moreover, if $\mathcal{T}_{1}=\left.\mathcal{S}_{1}\right|_{0_{\ddagger} \mathcal{H}_{1}}$ and $\mathcal{T}_{2}=\left.\mathcal{S}_{2}\right|_{I_{-} \mathcal{H}_{2}}$, then $\mathcal{T}_{1}$ and $\mathcal{T}_{2}$ are the projection lattices of von Neumann algebras and

$$
\begin{aligned}
& \text { (i) } \operatorname{Alg} \mathcal{S}_{1}=\left\{\left(\begin{array}{cc}
A & 0 \\
C & D
\end{array}\right): A \in \mathcal{T}_{1}^{\prime}, C \in \mathcal{B}\left(0_{+}^{\perp} \mathcal{H}_{1}, 0_{+} \mathcal{H}_{1}\right), D \in \mathcal{B}\left(0_{+} \mathcal{H}_{1}, 0_{+} \mathcal{H}_{1}\right)\right\} \\
& \text { and }\left(\mathcal{U}^{*} \mathcal{U}\right)^{\prime \prime}=\left\{\left(\begin{array}{cc}
A & 0 \\
0 & \lambda I
\end{array}\right): A \in \mathcal{T}_{1}^{\prime}, \lambda \in \mathbb{C}\right\}
\end{aligned}
$$


(ii) $\operatorname{Alg} \mathcal{S}_{2}=\left\{\left(\begin{array}{cc}B & C \\ 0 & D\end{array}\right): B \in \mathcal{T}_{2}^{\prime}, C \in \mathcal{B}\left(I_{-}^{\perp} \mathcal{H}_{2}, I_{-} \mathcal{H}_{2}\right), D \in \mathcal{B}\left(I_{-}^{\perp} \mathcal{H}_{2}, I_{-}^{\perp} \mathcal{H}_{2}\right)\right\}$ and $\left(\mathcal{U U}^{*}\right)^{\prime \prime}=\left\{\left(\begin{array}{cc}B & 0 \\ 0 & \lambda I\end{array}\right): B \in \mathcal{T}_{2}^{\prime}, \lambda \in \mathbb{C}\right\}$.

(iii) $\operatorname{Lat}\left(\mathcal{U}^{*} \mathcal{U}\right)=\left\{L \in \mathcal{P}_{1}: \chi(L) \perp \chi\left(L^{\perp}\right)\right\}=\left\{L_{1} \oplus L_{2}: L_{1} \in \mathcal{T}_{1}\right\}$.

Proof. Put $\chi^{*}=$ Map $\mathcal{U}^{*}$. Recall that $\mathcal{S}_{1}=\left\{P^{\perp}: P \in \chi^{*}\left(\mathcal{P}_{2}\right)\right\}$ and $\mathcal{S}_{2}=\chi\left(\mathcal{P}_{1}\right)$. First assume that $\mathcal{U}$ is essential. Let $\mathcal{X}=\operatorname{Lat}\left(\mathcal{U}^{*} \mathcal{U}\right)$ and $\mathcal{Y}=\operatorname{Lat}\left(\mathcal{U U}^{*}\right)$. Since $\mathcal{U}^{*} \mathcal{U}$ and $\mathcal{U} \mathcal{U}^{*}$ are selfadjoint sets of operators, $\mathcal{X}$ and $\mathcal{Y}$ are complete orthocomplemented projection lattices.

We will show that $\chi$ is an ortho-isomorphism from $\mathcal{X}$ onto $\mathcal{Y}$.

First, if $L \in \mathcal{X}$, then given $T, S \in \mathcal{U}$ we have $\left(T S^{*}\right)\left(R\left(L \mathcal{H}_{1}\right)\right)=\left(T S^{*} R\right)\left(L \mathcal{H}_{1}\right) \subseteq$ $\chi(L) \mathcal{H}_{2}$ for all $R \in \mathcal{U}$. Hence $\left(T S^{*}\right)\left(\chi(L) \mathcal{H}_{2}\right) \subseteq \chi(L) \mathcal{H}_{2}$ and so $\chi(L) \in \mathcal{Y}$. Similarly, $\chi^{*}(\mathcal{Y}) \subseteq \mathcal{X}$.

Next, we show that, if $L \in \mathcal{X}$, then $\chi^{*} \chi(L)=L$. For $T, S \in \mathcal{U}$ we have $\left(T^{*} S\right) L=$ $L\left(T^{*} S\right)$ since $L \in\left(\mathcal{U}^{*} \mathcal{U}\right)^{\prime}$ and so $L^{\perp}\left(T^{*} S\right) L=0$ and $L\left(T^{*} S\right) L^{\perp}=0$. The first relation gives $L^{\perp} T^{*}(\chi(L))=0$ for each $T \in \mathcal{U}$ and so $\chi^{*} \chi(L) \leq L$. Similarly the second gives $\chi^{*} \chi\left(L^{\perp}\right) \leq L^{\perp}$. But $\chi^{*} \chi\left(L \vee L^{\perp}\right)=\chi^{*} \chi(I)=I$ and so $\chi^{*} \chi(L) \vee$ $\chi^{*} \chi\left(L^{\perp}\right)=I$. The relation $\chi^{*} \chi(L)=L$ now follows readily. Applying the same arguments to the space $\mathcal{U}^{*}$, it follows that, if $M \in \mathcal{Y}$, then $\chi \chi^{*}(M)=M$.

So we have shown that $\chi$ maps $\mathcal{X}$ bijectively to $\mathcal{Y}$ and its inverse is $\chi^{*}$. But $\chi$ preserves orthogonality (indeed if $T, S \in \mathcal{U}$ and $L \in \mathcal{X}$ then $\left\langle T L x, S L^{\perp} y\right\rangle=$ $\left\langle S^{*} T L x, L^{\perp} y\right\rangle=0$ since $\left.L^{\perp} S^{*} T L=0\right)$ and since it is $\vee$-continuous and preserves 0 and $I$, it follows that it is $\wedge$-continuous as well and hence an ortho-isomorphism.

Finally, notice that $\mathcal{S}_{2}=\mathcal{Y}$ and $\mathcal{S}_{1}=\mathcal{X}$. Indeed, given $P \in \mathcal{P}_{1}$ and $T, S \in \mathcal{U}$ we have $\chi(P)^{\perp}\left(T S^{*}\right) R P=0$ for all $R \in \mathcal{U}$ (since $T S^{*} R \in \mathcal{U}$ and $\overline{[\mathcal{U}(P)]}=\chi(P)$ ), hence $\chi(P)^{\perp}\left(T S^{*}\right) \chi(P)=0$. Thus $\chi(P) \in \mathcal{Y}$, for each $P \in \mathcal{P}_{1}$ and so $\mathcal{S}_{2 \chi}=\chi\left(\mathcal{P}_{1}\right) \subseteq \mathcal{Y}$. Also if $M \in \mathcal{Y}$ then $M=\chi(L) \in \mathcal{S}_{2 \chi}$ where $L=\chi^{*}(M)$. This shows that $\mathcal{Y}=\mathcal{S}_{2}$. The proof that $\mathcal{X}=\mathcal{S}_{1}$ is similar.

Now relax the assumption that $\mathcal{U}$ is essential. Note that $0_{+}^{\perp}$ acts as the identity on $\mathcal{U}^{*} \mathcal{U}$ and hence $0_{+} \in\left(\mathcal{U}^{*} \mathcal{U}\right)^{\prime \prime}$. Similarly $I_{-} \in\left(\mathcal{U U}^{*}\right)^{\prime \prime}$ acts as the identity on $\mathcal{U U}^{*}$ and $\mathcal{U}\left(\mathcal{H}_{1}\right)=\mathcal{U}\left(0_{+}^{\perp} \mathcal{H}_{1}\right) \subseteq I_{-} \mathcal{H}_{2}$. Let $\mathcal{K}_{1}=0_{+}^{\perp} \mathcal{H}_{1}, \mathcal{K}_{2}=I_{-} \mathcal{H}_{2}$ and $\mathcal{U}_{o}=\left.\mathcal{U}\right|_{\mathcal{K}_{1}} \subseteq$ $\mathcal{B}\left(\mathcal{K}_{1}, \mathcal{K}_{2}\right)$. Then $\mathcal{U}_{o}$ is normalizing and essential. If $\chi_{o}$ is the map of $\mathcal{U}_{o}$ then clearly its semilattices are $\mathcal{S}_{1 \chi_{o}}=\left.\mathcal{S}_{1 \chi}\right|_{\mathcal{K}_{1}}=\mathcal{T}_{1}$ and $\mathcal{S}_{2 \chi_{o}}=\mathcal{S}_{2 \chi} \mid \mathcal{K}_{2}=\mathcal{T}_{2}$. By what was shown above, $\mathcal{T}_{1}=\operatorname{Lat}\left(\mathcal{U}_{o}^{*} \mathcal{U}_{o}\right), \mathcal{T}_{2}=\operatorname{Lat}\left(\mathcal{U}_{o} \mathcal{U}_{o}^{*}\right)$ and $\chi_{o}: \mathcal{T}_{1} \rightarrow \mathcal{T}_{2}$ is an isomorphism. Also $\left(\mathcal{U}^{*} \mathcal{U}\right)^{\prime \prime}=\overline{\left[\mathcal{U}_{o}^{*} \mathcal{U}_{o}\right]} \oplus \mathbb{C} I_{\mathcal{K}_{1}}$ and $\left(\mathcal{U} \mathcal{U}^{*}\right)^{\prime \prime}=\overline{\left[\mathcal{U}_{o} \mathcal{U}_{o}^{*}\right]} \oplus \mathbb{C} I_{\mathcal{K}_{2}}$.

It follows that $\operatorname{Lat}\left(\mathcal{U}^{*} \mathcal{U}\right)=\left\{L_{1} \oplus L_{2}: L_{1} \in \mathcal{T}_{1}\right\}$, while the other equality of (iii) is readily verified, in fact for every subspace $\mathcal{U}$ with map $\chi$.

Now a projection $M \in \mathcal{P}_{2}$ is in $\mathcal{S}_{2 \chi}=\chi\left(\mathcal{P}_{1}\right)$ if and only if it is of the form $M=M_{1} \oplus 0$ with respect to the decomposition $\mathcal{H}_{2}=\mathcal{K}_{2} \oplus \mathcal{K}_{2}^{\perp}$, where $M_{1} \in \mathcal{S}_{2 \chi_{o}}$. Similarly working with $\mathcal{U}^{*}$ we obtain that $\chi^{*}\left(\mathcal{P}_{2}\right)=\left\{L_{1} \oplus 0: L_{1} \in \mathcal{S}_{1 \chi_{o}}\right\}$ with respect to the decomposition $\mathcal{H}_{1}=\mathcal{K}_{1} \oplus \mathcal{K}_{1}^{\perp}$. Thus

$$
\mathcal{S}_{1 \chi}=\left\{L_{1} \oplus I: L_{1} \in \mathcal{T}_{1}\right\}, \quad \mathcal{S}_{2 \chi}=\left\{M_{1} \oplus 0: M_{1} \in \mathcal{T}_{2}\right\}
$$

From this we see that $X \in \operatorname{Alg} \mathcal{S}_{1 \chi}$ if and only if $\left(L_{1} \oplus I\right)^{\perp} X\left(L_{1} \oplus I\right)=0$ for each $L_{1} \in \mathcal{T}_{1}$, and claim (i) follows easily. The proof of (ii) is similar. 
Remarks (i) It is not difficult to show that the von Neumann algebras $\mathcal{T}_{1}^{\prime \prime}$ and $\mathcal{T}_{2}^{\prime \prime}$ are in fact ${ }^{*}$-isomorphic, and so the strongly closed algebras generated by $\mathcal{U}^{*} \mathcal{U}$ and $\mathcal{U U}^{*}$ are Morita equivalent [14]. We have preferred the direct approach above, which is sufficient for our needs.

(ii) The first part of the above theorem remains valid even if $\chi$ is not given as a map of some normalizing subspace, but is an arbitrary ortho-map. That is, the following lattice-theoretic result holds: If $\chi$ is an ortho-map, then its semi-lattices $\mathcal{S}_{1}$ and $\mathcal{S}_{2}$ are in fact complete ortho-lattices and $\chi: \mathcal{S}_{1} \longrightarrow \mathcal{S}_{2}$ is a complete orthoisomorphism [16].

The next corollary shows that the semilattices of the map of a normalizing space become reflexive lattices, if the extreme elements 0 and $I$ are adjoined.

Corollary 2.11. Suppose that $\mathcal{S}_{1}$ and $\mathcal{S}_{2}$ are the (semi-)lattices of the map $\chi$ of some normalizing subspace. Then $\operatorname{Lat} \operatorname{Alg} \mathcal{S}_{1}=\mathcal{S}_{1} \cup\{0\}$ and Lat $\operatorname{Alg} \mathcal{S}_{2}=\mathcal{S}_{2} \cup\{I\}$. Moreover, in the notation of Theorem 2.10, the orthocomplemented projection lattice generated by $\mathcal{S}_{1}$, is $\mathcal{S}_{1} \cup\left\{P_{1} \oplus 0: P_{1} \in \mathcal{T}_{1}\right\}$, while the orthocomplemented projection lattice generated by $\mathcal{S}_{2}$ is $\mathcal{S}_{2} \cup\left\{Q_{1} \oplus I: Q_{1} \in \mathcal{T}_{2}\right\}$.

Proof. $\quad$ Let $\Sigma_{1}=\operatorname{Lat} \operatorname{Alg} \mathcal{S}_{1}, \Sigma_{2}=\operatorname{Lat} \operatorname{Alg} \mathcal{S}_{2}$ and $\widetilde{\Sigma_{1}}$ and $\widetilde{\Sigma_{2}}$ be the orthocomplemented lattices generated by $\Sigma_{1}$ and $\Sigma_{2}$ respectively. Suppose that a projection $Q$ is left invariant by the algebra $\operatorname{Alg} \mathcal{S}_{1}$. Then by Theorem 2.10 it must be contained in $\operatorname{Lat}\left(\mathcal{U}^{*} \mathcal{U}\right)$; thus $Q$ is of the form $Q=Q_{1} \oplus Q_{2}$, where $Q_{1} \in \mathcal{T}_{1}$. Since $\left.0_{+} \operatorname{Alg} \mathcal{S}_{1}\right|_{0_{+}} \mathcal{H}_{1}=\mathcal{B}\left(0_{+} \mathcal{H}_{1}, 0_{+} \mathcal{H}_{1}\right)$, it follows that either $Q_{2}=I$ or $Q_{2}=0$. If $Q_{2}=I$ then $Q \in \mathcal{S}_{1}$. If $Q_{2}=0$, then $C Q_{1}=0$ for each $C \in \mathcal{B}\left(0_{+}^{\perp} \mathcal{H}_{1}, 0_{+} \mathcal{H}_{2}\right)$ and it follows that $Q_{1}=0$. Thus we have that $\Sigma_{1}=\mathcal{S}_{1} \cup\{0\}$.

It is easy to check that the set $\mathcal{S}_{1} \cup\left\{Q_{1} \oplus 0: Q_{1} \in \mathcal{T}_{1}\right\}$ is an orthocomplemented lattice. Since it contains $\mathcal{S}_{1}$, it must equal $\widetilde{\Sigma_{1}}$.

The other identities are proved by the same arguments. $\diamond$

Recall that a subspace $\mathcal{U}$ is called strongly reflexive [5], if there is a set of rank one operators $\mathcal{R}$ such that $\mathcal{U}=\operatorname{Ref} \mathcal{R}$. We wish to describe the strongly reflexive normalizing subspaces. The unital algebra case might be known; we include a proof for completeness.

Lemma 2.12. Every strongly reflexive selfadjoint unital algebra contains a totally atomic maximal abelian selfadjoint algebra (a masa).

Proof. If $\mathcal{A} \subseteq \mathcal{B}(\mathcal{H})$ is a strongly reflexive selfadjoint unital algebra, then it is a von Neumann algebra, since it is reflexive. If $\mathcal{L}=$ Lat $\mathcal{A}$, then $\mathcal{L}=$ Lat $\mathcal{R}$, where $\mathcal{R} \subseteq \mathcal{A}$ is the rank one subalgebra of $\mathcal{A}$, by strong reflexivity. It follows from [11] that $\mathcal{L}$ is completely distributive. But $\mathcal{L}$ is orthocomplemented, hence, by Tarski's theorem (see [2], p.119), it must be a complete atomic Boolean lattice. Thus, as is well-known, $\mathcal{L}$ is commutative, hence $\mathcal{L} \subseteq \mathcal{A}$. Therefore there exists a totally atomic masa $\mathcal{M}$ with $\mathcal{L} \subseteq \mathcal{M} \subseteq \mathcal{A}$.

Proposition 2.13. Every strongly reflexive normalizing subspace $\mathcal{U} \subseteq \mathcal{B}\left(\mathcal{H}_{1}, \mathcal{H}_{2}\right)$ is a bimodule over two totally atomic masas.

Proof. Let $x \in \overline{\mathcal{U}^{*} \mathcal{H}_{2}} \equiv \mathcal{K}_{1}$. If $S, T \in \mathcal{U}$, then, since $\mathcal{U}$ is strongly reflexive, $S^{*} T x$ can be approximated by vectors of the form $S^{*} R x$, where $R$ belongs to the rank one 
subspace of $\mathcal{U}$. The strong closure of the linear span of $\left.\mathcal{U}^{*} \mathcal{U}\right|_{\mathcal{K}_{1}}$ is therefore a strongly reflexive von Neumann algebra acting on the space $\mathcal{K}_{1}$. Thus it must contain a totally atomic masa by Lemma 2.12. Since $\mathcal{B}\left(\mathcal{K}_{1}^{\perp}, \mathcal{K}_{1}^{\perp}\right)$ also contains a totally atomic masa, it follows from Theorem 2.10 that $\operatorname{Alg} \mathcal{S}_{1}$ contains a totally atomic masa.

The proof that $\operatorname{Alg} \mathcal{S}_{2}$ contains a totally atomic masa is identical. Since $\mathcal{U}$ is an $\left(\operatorname{Alg} \mathcal{S}_{2}, \operatorname{Alg} \mathcal{S}_{1}\right)$-bimodule, we are done.

We will in fact prove (Theorem 3.5) that normalizing strongly reflexive subspaces are of the form $\oplus_{\lambda} \mathcal{B}\left(\mathcal{H}_{\lambda}, \mathcal{K}_{\lambda}\right)$, for some mutually orthogonal families of subspaces $\mathcal{H}_{\lambda} \subseteq \mathcal{H}_{1}$ and $\mathcal{K}_{\lambda} \subseteq \mathcal{H}_{2}$.

\section{NORMALIZING MASA BIMOdULES}

In this and the next section, we specialize to subspaces $\mathcal{U} \subseteq \mathcal{B}\left(\mathcal{H}_{1}, \mathcal{H}_{2}\right)$ which are bimodules over maximal abelian selfadjoint algebras (for short masas) $\mathcal{D}_{1} \subseteq \mathcal{B}\left(\mathcal{H}_{1}\right)$ and $\mathcal{D}_{2} \subseteq \mathcal{B}\left(\mathcal{H}_{2}\right)$ in the sense that $\mathcal{D}_{2} \mathcal{U} \mathcal{D}_{1} \subseteq \mathcal{U}$. As we will be using measure-theoretic arguments, we will make the blanket assumption that from now on all Hilbert spaces will be separable. If $\mathcal{L}_{1}$ and $\mathcal{L}_{2}$ are complete projection lattices, we let

$$
\mathcal{M}\left(\mathcal{L}_{1}, \mathcal{L}_{2}\right)=\left\{\varphi \in \mathcal{M}\left(\mathcal{P}_{1}, \mathcal{P}_{2}\right): \mathcal{S}_{i \varphi} \subseteq \mathcal{L}_{i}, i=1,2\right\}
$$

Theorem 3.1. A $w^{*}$-closed masa-bimodule $\mathcal{U}$ is normalizing if and only if there exist nests $\mathcal{N}_{1}, \mathcal{N}_{2}$ and a map $\varphi \in \mathcal{M}\left(\mathcal{N}_{1}, \mathcal{N}_{2}\right)$ such that $\mathcal{U}=\operatorname{Op}(\varphi) \cap \operatorname{Op}\left(\varphi^{\perp}\right)$.

Proof. Every space of the form $\mathcal{U}=\mathrm{Op}(\varphi) \cap \mathrm{Op}\left(\varphi^{\perp}\right)$ is normalizing (Theorem 2.4); and if $\varphi$ is a nest map, then $\mathcal{U}$ is a masa bimodule.

For the converse, write $\mathcal{U}$ as $\mathrm{Op} \chi$ for an ortho-map $\chi$. Recall (Theorem 2.10) that the lattice $\mathcal{S}_{1 o}=\left.\mathcal{S}_{1 \chi}\right|_{\left(0_{+} \mathcal{H}_{1}\right)^{\perp}}$ is orthocomplemented, hence, since $\mathcal{S}_{1 \chi} \subseteq \mathcal{D}_{1}$, a (complete) Boolean lattice. There exists a complete nest $\mathcal{N}_{o} \subseteq \mathcal{S}_{1 o}$ on the space $\left(0_{+} \mathcal{H}_{1}\right)^{\perp}$, generating $\mathcal{S}_{1 o}$ as a complete Boolean lattice [1]. Then the nest $\mathcal{N}_{1}=$ $\left\{Q \oplus I: Q \in \mathcal{N}_{o}\right\} \cup\{0\}$ on $\mathcal{H}_{1}$ will generate the complete Boolean lattice $\widetilde{\Sigma_{1}}$ generated by $\mathcal{S}_{1 \chi}$. We define the map $\varphi$ to be the restriction of $\chi$ to $\mathcal{N}_{1}$. From the fact that $\mathcal{N}_{1}$ is contained in $\mathcal{S}_{1 \chi} \cup\{0\}$ it is clear that the left semi-lattice of $\varphi, \mathcal{S}_{1 \varphi}$, equals $\mathcal{N}_{1} \backslash\{0\}$ (or $\mathcal{N}_{1}$, if $0_{+}=0$ ). If $X \in \mathrm{Op}(\chi)$, then certainly $X \in \mathrm{Op}(\varphi) \cap \mathrm{Op}\left(\varphi^{\perp}\right)$. Conversely, let $X \in \operatorname{Op}(\varphi) \cap \operatorname{Op}\left(\varphi^{\perp}\right)$. For each $N \in \mathcal{N}_{1}$, we have $X N \mathcal{H}_{1} \subseteq \varphi(N) \mathcal{H}_{2}=\chi(N) \mathcal{H}_{2}$ and $X N^{\perp} \mathcal{H}_{1} \subseteq \varphi(I) \varphi(N)^{\perp} \mathcal{H}_{2}=\chi(I) \chi(N)^{\perp} \mathcal{H}_{2}=\chi\left(N^{\perp}\right) \mathcal{H}_{2}$. Because $\mathcal{N}_{1}$ generates $\widetilde{\Sigma_{1}}$ and $\chi$ preserves arbitrary unions and intersections, it follows that $X \in \operatorname{Op} \chi . \diamond$

To state the next results, we need to recall some terminology from [5]. Let $(X, \mu)$, $(Y, \nu)$ be standard Borel spaces and let $\mathcal{D}_{1}, \mathcal{D}_{2}$ be the multiplication masas on the corresponding $L^{2}$ spaces $\mathcal{H}_{1}, \mathcal{H}_{2}$, with projections denoted by $E(\alpha) \in \mathcal{D}_{1}$ and $F(\beta) \in$ $\mathcal{D}_{2}$, where $\alpha \subseteq X$ and $\beta \subseteq Y$ are Borel sets. A bounded operator $T: \mathcal{H}_{1} \rightarrow \mathcal{H}_{2}$ is said to be supported by a set $\kappa \subseteq X \times Y$ if $F(\beta) T E(\alpha)=0$ whenever $(\alpha \times \beta) \cap \kappa=\emptyset$. It is shown in [5] that a masa bimodule $\mathcal{U}$ is reflexive precisely when there exists a set $\kappa \subseteq X \times Y$ such that $\mathcal{U}$ consists of all operators supported by $\kappa$. This set is uniquely defined up to marginal equivalence, and is called the $\omega$-support of $\mathcal{U}$; its complement is (marginally equivalent to) a countable union of Borel rectangles.

Theorem 3.2. Let $\mathcal{H}_{1}=L^{2}(X, \mu), \mathcal{H}_{2}=L^{2}(Y, \nu)$ and let $\mathcal{U} \subseteq \mathcal{B}\left(\mathcal{H}_{1}, \mathcal{H}_{2}\right)$ be a $w^{*}$ closed normalizing space which is a bimodule over the multiplication masas $\mathcal{D}_{i} \subseteq$ 
$\mathcal{B}\left(\mathcal{H}_{i}\right)$. Then there exist Borel functions $f: X \rightarrow[0,1]$ and $g: Y \rightarrow[0,1]$ such that the $\omega$-support of $\mathcal{U}$ is the set $\kappa=\{(x, y) \in X \times Y: f(x)=g(y)\}$.

Conversely, the set of all operators supported by a set of the above form is a $w^{*}$ closed normalizing masa bimodule.

Proof Let $\chi$ be the map of $\mathcal{U}$, and let $X_{o} \subseteq X$ and $Y_{o} \subseteq Y$ be Borel sets such that $E\left(X_{o}\right)=\overline{\left[\mathcal{U}^{*} \mathcal{H}_{2}\right]}$ and $F\left(Y_{o}\right)=\overline{\left[\mathcal{U H}_{1}\right]}$. Theorem 2.10 shows that $\mathcal{S}_{1 o}=\left.\mathcal{S}_{1 \chi}\right|_{E\left(X_{o}\right)}$ and $\mathcal{S}_{2 o}=\left.\mathcal{S}_{2 \chi}\right|_{F\left(Y_{o}\right)}$ are complete orthocomplemented lattices, hence (since they are commutative) complete Boolean lattices, and that $\chi$ induces a complete Boolean lattice isomorphism $\chi_{o}$ between them. Denote by $\mathfrak{A}_{i}$ the $\mathrm{w}^{*}$-closed algebra generated by $\mathcal{S}_{i o}(i=1,2)$, and let $\psi: \mathfrak{A}_{1} \rightarrow \mathfrak{A}_{2}$ be the ${ }^{*}$-isomorphism induced by $\chi_{o}$. Now the $\mathfrak{A}_{i}$ are abelian separably acting von Neumann algebras, hence there exist standard Borel probability spaces $\left(X_{1}, \mu_{1}\right)$ and $\left(Y_{1}, \nu_{1}\right)$ such that the $\mathfrak{A}_{i}$ are ${ }^{*}$-isomorphic to the corresponding $L^{\infty}$ spaces. Moreover, we can take both $X_{1}$ and $Y_{1}$ to be compact intervals, which we take to be $\left[\frac{1}{3}, \frac{2}{3}\right]$ for notational convenience. Now the inclusion $\mathfrak{A}_{1} \rightarrow \mathcal{D}_{1}$ maps the identity of $\mathfrak{A}_{1}$ to $E\left(X_{o}\right)$, and hence induces an injective unital *-homomorphism $\theta_{1}: L^{\infty}\left(X_{1}, \mu_{1}\right) \rightarrow L^{\infty}\left(X_{o}, \mu\right)$. This map is implemented (see [15]) by a Borel function $f_{1}: X_{o} \rightarrow X_{1}$; thus every projection $E \in \mathfrak{A}_{1}$ is of the form $E=E\left(f_{1}^{-1}(\sigma)\right)$ for some Borel subset $\sigma \subseteq X_{1}$. Since $\theta_{1}$ is injective, $f_{1}$ can be taken to be onto $X_{1}$. Similarly, there exists a Borel onto function $g_{1}: Y_{o} \rightarrow Y_{1}$ such that every projection $F \in \mathfrak{A}_{2}$ is of the form $F=F\left(g_{1}^{-1}(\tau)\right)$ for some Borel subset $\tau \subseteq Y_{1}$. Now the ${ }^{*}$-isomorphism $\psi: \mathfrak{A}_{1} \rightarrow \mathfrak{A}_{2}$ is also implemented by a Borel bijection $h: Y_{1} \rightarrow X_{1}$; that is, for every projection $E\left(f_{1}^{-1}(\sigma)\right)$ we have $\psi\left(E\left(f_{1}^{-1}(\sigma)\right)\right)=F\left(g_{1}^{-1}\left(h^{-1}(\sigma)\right)\right)$. Define $f: X \rightarrow[0,1]$ by $f(x)=f_{1}(x)$ for $x \in X_{o}$ and $f(x)=0$ otherwise, and define $g: Y \rightarrow[0,1]$ by $g(y)=h\left(g_{1}(y)\right)$ for $y \in Y_{o}$ and $g(y)=1$ otherwise. Thus $\mathcal{S}_{1 \chi}=\left\{E\left(f^{-1}(\sigma)\right): \sigma \subseteq[0,1]\right.$ Borel, $\left.0 \in \sigma\right\}$ and $\chi\left(E\left(f^{-1}(\sigma)\right)=F\left(g^{-1}(\sigma)\right)\right.$.

Let $\kappa=\{(x, y) \in X \times Y: f(x)=g(y)\}$. Pick a countable dense subset $\left\{s_{n}\right\} \subseteq[0,1)$ and note that $\kappa=\left\{(x, y) \in X \times Y\right.$ : for each $\left.n, x \in \alpha_{n} \Leftrightarrow y \in \beta_{n}\right\}$ where $\alpha_{n}=$ $f^{-1}\left(\left[0, s_{n}\right]\right)$ and $\beta_{n}=g^{-1}\left(\left[0, s_{n}\right]\right)$, so that $\chi\left(E\left(\alpha_{n}\right)\right)=F\left(\beta_{n}\right)$ and $\chi\left(E\left(\alpha_{n}^{c}\right)\right) \subseteq F\left(\beta_{n}^{c}\right)$. Thus, the complement of $\kappa$ can be written $\kappa^{c}=\cup_{k} \gamma_{k} \times \delta_{k}$ where $\chi\left(E\left(\gamma_{k}\right)\right) \subseteq F\left(\delta_{k}^{c}\right)$ for each $k$.

It is shown in the proof of Theorem 4.2 of [5] that an operator $T$ is supported by $\kappa$ if and only if $F\left(\delta_{k}\right) T E\left(\gamma_{k}\right)=0$ for all $k \in \mathbb{N}$. Thus if $T \in \mathcal{U}$ then $T$ is supported by $\kappa$.

Conversely, suppose $T$ is supported by $\kappa$. Then for each $E=E\left(f^{-1}(\sigma)\right) \in \mathcal{S}_{1 \chi}$ we have $\chi(E)^{\perp} T E=F\left(g^{-1}(\sigma)^{c}\right) T E\left(f^{-1}(\sigma)\right)$ since $\chi(E)=F\left(g^{-1}(\sigma)\right)$. But the rectangle $f^{-1}(\sigma) \times g^{-1}(\sigma)^{c}$ is disjoint from $\kappa$, hence $\chi(E)^{\perp} T E=0$ so that $T \in \mathcal{U}$.

Finally, if $\mathcal{U}$ consists of all operators supported by a set $\kappa$ of the above form, then writing $\kappa=\left\{(x, y) \in X \times Y\right.$ : for each $\left.n, x \in \alpha_{n} \Leftrightarrow y \in \beta_{n}\right\}$ as above we have that an operator $T$ is in $\mathcal{U}$ if and only if $F\left(\beta_{n}\right)^{\perp} T E\left(\alpha_{n}\right)=0$ and $F\left(\beta_{n}\right) T E\left(\alpha_{n}\right)^{\perp}=0$ for each $n$. Thus $\mathcal{U}=\left\{T \in \mathcal{B}\left(\mathcal{H}_{1}, \mathcal{H}_{2}\right): T E\left(\alpha_{n}\right)=F\left(\beta_{n}\right) T\right.$ for each $\left.n\right\}$, and this is clearly closed under the triple product. $\diamond$

Corollary 3.3. In the notation of the last Theorem, if the algebra $\left[\mathcal{U U}^{*}\right]$ (resp. $\left.\left[\mathcal{U}^{*} \mathcal{U}\right]\right)$ is abelian, then the $\omega$-support of $\mathcal{U}$ is the graph (resp. 'reverse graph') of a Borel function $f: X_{o} \rightarrow Y$ (resp. $g: Y_{o} \rightarrow X$ ) for a suitable Borel subset $X_{o} \subseteq X$ (resp. $Y_{o} \subseteq Y$ ). 
Proof If $\left[\mathcal{U}^{*} \mathcal{U}\right]$ is abelian, then its $\mathrm{w}^{*}$-closure is maximal abelian on $\overline{\left[\mathcal{U}^{*} \mathcal{H}_{2}\right]}$. Hence it must be unitarily equivalent to the multiplication algebra of $L^{\infty}\left(X_{o}, \mu\right)$. It follows as in the above proof (essentially by replacing $f_{1}$ by the identity $X_{o} \rightarrow X_{o}$ ) that there exists a Borel function $g: Y_{o} \rightarrow X$ such that the $\omega$-support of $\mathcal{U}$ is $\{(x, y) \in$ $\left.X \times Y_{o}: x=g(y)\right\}$.

Similarly, if $\left[\mathcal{U U}^{*}\right]$ is abelian, there exists a Borel function $f: X_{o} \rightarrow Y$ such that the $\omega$-support of $\mathcal{U}$ is $\left\{(x, y) \in X_{o} \times Y: f(x)=y\right\} . \diamond$

Remarks (i) The previous Theorem is based on an idea of V.S. Shulman. We take this opportunity to thank him.

(ii) Based on the characterization of Theorem 3.1 a constructive proof of Theorem 3.2 can be given, avoiding the use of the implementability of ${ }^{*}$-homomorphisms between $L^{\infty}$-spaces (see [16]).

(iii) In general, one function is not enough to describe the support of a normalizing masa-bimodule. For an example, consider the von Neumann algebra $\mathcal{A}=M_{2}(\mathcal{M})$, where $\mathcal{M}$ is the multiplication algebra of $L^{\infty}(0,1)$. It is not hard to see that the support of $\mathcal{A}$ is a set of the form $\{(x, y): f(x)=f(y)\}$, where $f$ is a certain Borel function on $[0,2]$ with period 1 .

It is easy to see that every finite rank operator in a von Neumann algebra with abelian commutant is a sum of rank one operators in the algebra. Hence, for normalizing masa bimodules Corollary 2.7 can be improved as follows:

Proposition 3.4. Let $\mathcal{U}$ be an ultraweakly closed normalizing masa bimodule and suppose that $K$ is a compact operator in $\mathcal{U}$. Then $K$ can be approximated in the norm topology by sums of rank one operators in $\mathcal{U}$. Moreover, if $K \in \mathcal{C}_{p}$ then it can be approximated by sums of rank one operators in $\mathcal{U}$ in the p-norm topology. Finally, every operator of rank $n$ in $\mathcal{U}$ is the sum of $n$ rank one operators in $\mathcal{U}$.

Next we want to identify the normalizing masa-bimodules which are strongly reflexive. If a rank one operator $R$ belongs to the masa-bimodule $\mathcal{U}$ and if $E_{R}$ and $F_{R}$ are the smallest projections in the masas such that $F_{R} R=R$ and $R E_{R}=R$, then $F_{R} \mathcal{B}\left(\mathcal{H}_{1}, \mathcal{H}_{2}\right) E_{R} \subseteq \mathcal{U}$. It was proved in [5] that, if $\mathcal{U}$ is a strongly reflexive masa-bimodule, the space

$$
\operatorname{span}\left\{F_{R} \mathcal{B}\left(\mathcal{H}_{1}, \mathcal{H}_{2}\right) E_{R}: R \in \mathcal{U}\right\}
$$

is weakly dense in $\mathcal{U}$. Note that $\mathcal{B}\left(\mathcal{H}_{1}, \mathcal{H}_{2}\right)$ is a strongly reflexive normalizing masa bimodule. More generally, if $\left\{E_{n}\right\} \subseteq \mathcal{D}_{1}$ and $\left\{F_{n}\right\} \subseteq \mathcal{D}_{2}$ are countable families of mutually orthogonal projections, it is easy to verify that the direct sum $\oplus_{n} F_{n} \mathcal{B}\left(\mathcal{H}_{1}, \mathcal{H}_{2}\right) E_{n}$ is closed under the triple product, and is clearly a strongly reflexive normalizing masa bimodule. In fact, there are no others:

Theorem 3.5. A strongly reflexive subspace $\mathcal{U}$ is normalizing if and only if there are countable families $\left\{E_{n}\right\}$ and $\left\{F_{n}\right\}$ consisting of mutually orthogonal projections, such that

$$
\mathcal{U}=\oplus F_{n} \mathcal{B}\left(\mathcal{H}_{1}, \mathcal{H}_{2}\right) E_{n}
$$

Proof. Let $\mathcal{U}$ be a normalizing strongly reflexive subspace and let $\mathcal{U}_{o}$ be its essential part acting on $\mathcal{K}_{1}=0_{+}^{\perp} \mathcal{H}_{1}$. If $\mathcal{A} \subseteq \mathcal{B}\left(\mathcal{K}_{1}\right)$ is the von Neumann algebra $\left(\mathcal{U}_{o}^{*} \mathcal{U}_{o}\right)^{\prime \prime}$, we have shown (Lemma 2.12) that $\mathcal{L}=$ Lat $\mathcal{A}$ is a totally atomic commutative Boolean lattice. Let $\mathcal{E}=\left\{E_{n}: n=1,2, \ldots\right\}$ be the set of atoms of $\mathcal{L}$ considered as projections 
in $\mathcal{H}_{1}$ (necessarily countable since $\mathcal{H}_{1}$ is separable). Since $\sum E_{n}=0_{+}^{\perp}$ in the strong operator topology, each $T \in \mathcal{U}$ can be written $T=\sum T E_{n}$. Setting $F_{n}=\left[\mathcal{U} E_{n}\right]=$ $\chi\left(E_{n}\right)$ (where $\chi=\operatorname{Map}(\mathcal{U})$ ), we see that the $F_{n}$ are orthogonal since $\chi$ is an orthomap. Since $T E_{n}=F_{n} T E_{n}$, it follows that $T=\sum F_{n} T E_{n}$. On the other hand, for each $n$, each $T \in F_{n} \mathcal{B}\left(\mathcal{H}_{1}, \mathcal{H}_{2}\right) E_{n}$ satisfies $\chi(L)^{\perp} T L=0$ when $L$ is some $E_{m}$, and hence when $L$ is in $\mathcal{L}$. Thus $F_{n} \mathcal{B}\left(\mathcal{H}_{1}, \mathcal{H}_{2}\right) E_{n} \subseteq \mathcal{U}$ for each $n$, and this completes the proof. $\diamond$

The proof of the above theorem actually gives more.

Corollary 3.6. The ultraweak closure of the rank one subspace of a normalizing subspace $\mathcal{U} \subseteq \mathcal{B}(\mathcal{H}, \mathcal{K})$ is of the form $\oplus_{n} \mathcal{B}\left(\mathcal{H}_{n}, \mathcal{K}_{n}\right)$, for some mutually orthogonal families of subspaces $\mathcal{H}_{n} \subseteq \mathcal{H}$ and $\mathcal{K}_{n} \subseteq \mathcal{K}$.

Proof. Obviously the rank one subspace of $\mathcal{U}$ is also a normalizing space and hence its ultraweak closure is a reflexive (Theorem 2.4) normalizing masa bimodule. Thus the last theorem applies. $\diamond$

According to [5], a masa bimodule is strongly reflexive if and only if its $\omega$-support $\kappa$ is marginally equivalent to a countable union of Borel rectangles. In the terminology of [5], $\kappa$ is the $\omega$-closure of its $\omega$-interior. In this terminology, Corollary 3.6 says that the $\omega$-interior of the $\omega$-support of a normalizing masa-bimodule can be written as a countable union of Borel rectangles $\alpha_{n} \times \beta_{n}$, such that the families $\left\{\alpha_{n}\right\}$ and $\left\{\beta_{n}\right\}$ consist of disjoint Borel sets.

Let us recall that in [5] it was shown that although the rank one subspace of a general strongly reflexive masa-bimodule is dense in the bimodule in the weak operator topology, it need not be dense in the ultraweak topology. By Theorem 3.5, this cannot occur for normalizing masa-bimodules.

Corollary 3.7. If a normalizing subspace $\mathcal{U}$ is strongly reflexive, then its rank one subspace is ultraweakly dense in $\mathcal{U}$.

\section{NORMALIZING MASA-BIMODULES AND SYNTHESIS}

Now we turn our attention to the question of spectral synthesis. Spectral synthesis for operator algebras was introduced by Arveson [1]. It can be generalized for masabimodules as follows. If $\mathcal{L}_{1}=\mathcal{P}\left(\mathcal{D}_{1}\right), \mathcal{L}_{2}=\mathcal{P}\left(\mathcal{D}_{2}\right)$ are the projection lattices of two masas, a map $\varphi \in \mathcal{M}\left(\mathcal{L}_{1}, \mathcal{L}_{2}\right)$ (a commutative subspace map in the terminology of [6]) is said to be synthetic, if the only ultraweakly closed masa-bimodule $\mathcal{S}$ with the property $\operatorname{Map} \mathcal{S}=\varphi$ is $\operatorname{Op} \varphi$. There is a non trivial fact hidden behind this definition. As was proved by Arveson (in fact, for the case of CSL algebras, but it easily follows for masa-bimodules as well $\llbracket)$, given $\varphi \in \mathcal{M}\left(\mathcal{L}_{1}, \mathcal{L}_{2}\right)$ there exists an ultraweakly closed masa-bimodule $\mathcal{M}_{\text {min }}$, minimal with respect to the property that its reflexive hull equals $\operatorname{Op} \varphi$. Thus a reflexive masa-bimodule $\mathcal{M}$ has synthetic map if and only if $\mathcal{M}=\mathcal{M}_{\text {min }}$. Von Neumann algebras with abelian commutant have this property. The same holds for their generalization, normalizing masa-bimodules.

Theorem 4.1. Commutative subspace ortho-maps are synthetic.

This theorem will follow from a general fact about masa-bimodules (Proposition 4.2 below), which states that for every family of ultraweakly closed masa-bimodules with the same reflexive hull, there is a certain natural normalizing masa-bimodule 
contained in each member of the family. This bimodule corresponds to the diagonal of a CSL algebra.

Proposition 4.2. Let $\mathcal{U}=\mathrm{Op} \varphi \subseteq \mathcal{B}\left(\mathcal{H}_{1}, \mathcal{H}_{2}\right)$ be a reflexive masa bimodule, and let $\mathcal{U}_{0}=\operatorname{Op} \varphi \cap \mathrm{Op} \varphi^{\perp}$. Then $\mathcal{U}_{0}$ is contained in $\mathcal{U}_{\text {min }}$, the minimal ultraweakly closed masa-bimodule with reflexive cover $\mathcal{U}$.

Proof. We consider the Hilbert space $\mathcal{H}=\mathcal{H}_{2} \oplus \mathcal{H}_{1}$ and the set of projections

$$
\mathcal{S}=\left\{\varphi(L) \oplus L: L \in \mathcal{S}_{1 \varphi}\right\}
$$

on it. We put $\mathcal{A}=\operatorname{Alg} \mathcal{S}_{1 \varphi}, \mathcal{B}=\operatorname{Alg} \varphi\left(\mathcal{S}_{1 \varphi}\right)=\operatorname{Alg} \mathcal{S}_{2 \varphi}$ and $\mathcal{V}=\operatorname{Op} \varphi^{\perp}$.

It is an easy verification to show that $\operatorname{Alg} \mathcal{S}=\left(\begin{array}{cc}\mathcal{B} & \mathcal{U} \\ \mathcal{V}^{*} & \mathcal{A}\end{array}\right)$. A similar calculation shows also that the diagonal $\mathcal{C} \cap \mathcal{C}^{*}$ of $\mathcal{C}=\operatorname{Alg} \mathcal{S}$ (i.e. the commutant of $\mathcal{S}$ ) is $\mathcal{S}^{\prime}=\left(\begin{array}{cc}\mathcal{S}_{2 \varphi}^{\prime} & \mathcal{U}_{0} \\ \mathcal{U}_{0}^{*} & \mathcal{S}_{1 \varphi}^{\prime}\end{array}\right)$. But Arveson [1] has shown that, for a CSL algebra $\mathcal{C}$, the diagonal $\mathcal{C} \cap \mathcal{C}^{*}$ is contained in $\mathcal{C}_{\text {min. }}$. Therefore, to show that $\mathcal{U}_{0} \subseteq \mathcal{U}_{\text {min }}$, it suffices to prove that $\mathcal{C}_{\text {min }}$ is contained in

$$
\mathcal{M}=\left(\begin{array}{ll}
\mathcal{B}_{\min } & \mathcal{U}_{\text {min }} \\
\mathcal{V}_{\text {min }}^{*} & \mathcal{A}_{\text {min }}
\end{array}\right)
$$

Since $\mathcal{M}$ is a $\mathrm{w}^{*}$-closed subspace of $\mathcal{B}(H)$ containing the masa $\mathcal{D}_{2} \oplus \mathcal{D}_{1}$, to show that $\mathcal{C}_{\text {min }} \subseteq \mathcal{M}$ it suffices to show that $[\mathcal{M} \xi]=[\mathcal{C} \xi]$ for all $\xi \in \mathcal{H}$ (see Theorem 22.19 of [⿶] $)$. But this can be immediately verified using the facts that $\mathcal{B}=\operatorname{Ref}\left(\mathcal{B}_{\text {min }}\right)$, $\mathcal{U}=\operatorname{Ref}\left(\mathcal{U}_{\text {min }}\right), \mathcal{V}=\operatorname{Ref}\left(\mathcal{V}_{\text {min }}\right)$ and $\mathcal{A}=\operatorname{Ref}\left(\mathcal{A}_{\text {min }}\right)$.

Proof of Theorem 4.1. Suppose that $\chi$ is a commutative subspace map, which is also an ortho-map. The space $\mathcal{U}=\mathrm{Op} \chi$ is normalizing. But then $\mathcal{U}=\mathrm{Op} \chi \cap \mathrm{Op} \chi^{\perp}$ (Theorem 2.4), hence $\mathcal{U} \subseteq \mathcal{U}_{\text {min }}$ by Proposition 1.2. Thus $\mathcal{U}=\mathcal{U}_{\text {min }}$, which means that $\chi$ is synthetic. $\diamond$

Corollary 4.3. If the map of an ultraweakly closed masa-bimodule $\mathcal{U}$ is an orthomap, then $\mathcal{U}$ is normalizing.

Proof. Since $\mathcal{U}$ is a masa-bimodule, its map $\chi$ is commutative, hence by Theorem 4.1 it is synthetic. Hence $\mathcal{U}=\operatorname{Ref} \mathcal{U}=\mathrm{Op} \chi$. But since $\chi$ is an ortho-map, Op $\chi$ must be normalizing (Theorem 2.4). $\diamond$

We conclude this section with another result on synthesis. Let us call an ultraweakly closed masa bimodule synthetic if its map is synthetic. Thus a synthetic masa-bimodule is automatically reflexive. As we will easily see, normalizing masabimodules are not only synthetic, but in a sense hereditarily synthetic.

Proposition 4.4. Let $\mathcal{U}$ be an ultraweakly closed normalizing masa bimodule and let $\mathcal{S} \subseteq \mathcal{U}$ be an ultraweakly closed masa-bimodule. If $\mathcal{S}$ is a $\mathcal{U}^{*} \mathcal{U}$-submodule (or a $\mathcal{U U}^{*}$-submodule), then $\mathcal{S}$ is synthetic.

Proof. It suffices to show that $\mathcal{S}$ is normalizing. Let $\mathcal{U}=\operatorname{Op} \varphi, \mathcal{B}_{1}=\mathcal{U}^{*} \mathcal{U}$ and $\mathcal{B}_{2}=\mathcal{U U}^{*}$. Suppose that $R, S, T \in \mathcal{S}$. Then $S, T \in \mathcal{U}$ and hence $S^{*} T \in \mathcal{B}_{1}$. If $\mathcal{S B}_{1} \subseteq \mathcal{S}$, it follows that $R S^{*} T \in \mathcal{S}$. If $\mathcal{B}_{2} \mathcal{S} \subseteq \mathcal{S}$, we have $R S^{*} \in \mathcal{B}_{2}$ and hence $R S^{*} T \in \mathcal{S}$ again. $\diamond$ 
An immediate application of the last proposition is the fact that, if $\mathcal{A}$ is a von Neumann algebra with abelian commutant, every ultraweakly closed left or right ideal of $\mathcal{A}$ is synthetic. Note that this can also be inferred from the results of [12].

\section{Normalizers of REFleXive ALGEBras}

In this section we present the relation between normalizing reflexive subspaces and normalizers of reflexive algebras of operators. Let $\mathcal{A}$ and $\mathcal{B}$ be reflexive algebras of operators on $\mathcal{H}_{1}$ and $\mathcal{H}_{2}$ respectively. An operator $T \in \mathcal{B}\left(\mathcal{H}_{1}, \mathcal{H}_{2}\right)$ is called a semi-normalizer of $\mathcal{B}$ into $\mathcal{A}$ if

$$
T^{*} \mathcal{B} T \subseteq \mathcal{A} .
$$

A normalizer of the algebra $\mathcal{B}$ into the algebra $\mathcal{A}$ is a semi-normalizer of $\mathcal{B}$ into $\mathcal{A}$ whose adjoint is a semi-normalizer of $\mathcal{A}$ into $\mathcal{B}$. We denote by $S N(\mathcal{B}, \mathcal{A})$ the set of semi-normalizers of $\mathcal{B}$ into $\mathcal{A}$ and by $N(\mathcal{B}, \mathcal{A})$ the set of normalizers of $\mathcal{B}$ into $\mathcal{A}$.

In [3] it is shown that the set of normalizers of a nest algebra into itself is closed in the weak operator topology. We show that, for arbitrary reflexive algebras $\mathcal{A}$ and $\mathcal{B}$, the sets $S N(\mathcal{B}, \mathcal{A})$ and $N(\mathcal{B}, \mathcal{A})$ are closed in the strong operator topology, but not always in the weak operator topology; nevertheless, Coates' result remains valid whenever the algebras $\mathcal{A}$ and $\mathcal{B}$ are strongly reflexive.

Proposition 5.1. If $\mathcal{A}$ and $\mathcal{B}$ are reflexive algebras, the sets $S N(\mathcal{B}, \mathcal{A})$ and $N(\mathcal{B}, \mathcal{A})$ are strongly closed. If $\mathcal{B}$ is strongly reflexive (resp. $\mathcal{A}$ and $\mathcal{B}$ are strongly reflexive) then $S N(\mathcal{B}, \mathcal{A})$ (resp. $N(\mathcal{B}, \mathcal{A})$ ) is weakly closed. The sets $S N(\mathbb{C} I, \mathbb{C} I)$ and $N(\mathbb{C} I, \mathbb{C} I)$ are not weakly closed.

Proof. Suppose that $T_{\nu} \longrightarrow T$ strongly and that $T_{\nu} \in S N(\mathcal{B}, \mathcal{A})$. Then, for each operator $B \in \mathcal{B}$ and vectors $x$ and $y,\left\langle B T_{\nu} x, T_{\nu} y\right\rangle \longrightarrow\langle B T x, T y\rangle$. This means, of course, that $T_{\nu}^{*} B T_{\nu} \longrightarrow T^{*} B T$ in the weak operator topology. Since $T_{\nu}^{*} B T_{\nu} \in \mathcal{A}$ for each $\nu$ and $\mathcal{A}$ is weakly closed, being reflexive, it follows that $T^{*} B T \in \mathcal{A}$. The proof that $N(\mathcal{B}, \mathcal{A})$ is strongly closed is similar.

Now suppose that $T_{\nu} \longrightarrow T$ weakly and that $T_{\nu} \in S N(\mathcal{B}, \mathcal{A})$. First note that for each finite rank operator $F \in \mathcal{B}, T_{\nu}^{*} F T_{\nu} \longrightarrow T^{*} F T$ weakly (indeed, writing $F=F_{1}^{*} F_{2}$ where $F_{1}, F_{2}$ have finite rank, we have $F_{i} T_{\nu} \longrightarrow F_{i} T$ strongly for $i=1,2$ ). Thus $T^{*} F T \in \mathcal{A}$. Now if $B \in \mathcal{B}$ is arbitrary and $x \in \mathcal{H}_{2}$, by strong reflexivity there is a net $\left(F_{i}\right)$ of finite rank operators in $\mathcal{B}$ such that $F_{i} T x \rightarrow B T x$ and so $T^{*} F_{i} T x \rightarrow T^{*} B T x$. Since each $T^{*} F_{i} T$ is in $\mathcal{A}$ and $\mathcal{A}$ is reflexive, it follows that $T^{*} B T \in \mathcal{A}$. This shows that $S N(\mathcal{B}, \mathcal{A})$ is weakly closed. Therefore, if $\mathcal{A}$ is also strongly reflexive, the same holds for $N(\mathcal{B}, \mathcal{A})=S N(\mathcal{B}, \mathcal{A}) \cap(S N(\mathcal{A}, \mathcal{B}))^{*}$.

The final statement is a consequence of the fact that the sets of isometries and unitaries are not weakly closed. For a specific example, we construct a sequence $T_{n} \in N(\mathbb{C} I, \mathbb{C} I)$ converging weakly to an operator $T$ which is not in $S N(\mathbb{C} I, \mathbb{C} I)$. Let $\left\{e_{k}: k \in \mathbb{Z}\right\} \cup\{e\}$ be an orthonormal basis of $\mathcal{H}$ and let $U$ be the bilateral shift defined on $\mathcal{H}_{o}=\overline{\left[e_{k}: k \in \mathbb{Z}\right]}$ by $U e_{k}=e_{k+1}$. Define $T_{n}=U^{n} \oplus I \in \mathcal{B}(\mathcal{H})$ and $T=0 \oplus I \in \mathcal{B}(\mathcal{H})$. Each $T_{n}$ is a unitary operator on $\mathcal{H}$, and so $T_{n}^{*}(\mathbb{C} I) T_{n} \subseteq \mathbb{C} I$ and $T_{n}(\mathbb{C} I) T_{n}^{*} \subseteq \mathbb{C} I$, i.e. $T_{n} \in N(\mathbb{C} I, \mathbb{C} I)$. Since $U^{n} \rightarrow 0$ weakly, $T_{n} \rightarrow T$ weakly. But $T^{*}(\mathbb{C} I) T=[T] \nsubseteq \mathbb{C} I$.

Let $\mathcal{L}_{1}=$ Lat $\mathcal{A}$ and $\mathcal{L}_{2}=$ Lat $\mathcal{B}$. If $\varphi \in \mathcal{M}\left(\mathcal{L}_{1}, \mathcal{L}_{2}\right)$, we put

$$
\mathcal{U}_{\varphi}=\left\{T \in \mathcal{B}\left(\mathcal{H}_{1}, \mathcal{H}_{2}\right): T L=\varphi(L) T \text { for all } L \in \mathcal{L}_{1}\right\}
$$


which is a reflexive normalizing subspace, and also a bimodule over the diagonals $\mathcal{B} \cap \mathcal{B}^{*}$ and $\mathcal{A} \cap \mathcal{A}^{*}$, as is readily verified.

Theorem 5.2. The set $S N(\mathcal{B}, \mathcal{A})$ of semi-normalizers of $\mathcal{B}$ into $\mathcal{A}$ is a union of reflexive normalizing subspaces. More precisely,

$$
S N(\mathcal{B}, \mathcal{A})=\cup\left\{\mathcal{U}_{\varphi}: \varphi \in \mathcal{M}\left(\mathcal{L}_{1}, \mathcal{L}_{2}\right)\right\}
$$

Proof. Suppose that $\varphi \in \mathcal{M}\left(\mathcal{L}_{1}, \mathcal{L}_{2}\right)$ and $T \in \mathcal{U}_{\varphi}$. Then, for each $L \in \mathcal{L}_{1}$ and $B \in \mathcal{B}$,

$$
L^{\perp} T^{*} B T L=L^{\perp} T^{*} B \varphi(L) T=L^{\perp} T^{*} \varphi(L) B \varphi(L) T=L^{\perp} L T^{*} B \varphi(L) T=0
$$

since $\varphi(L) \in \mathcal{L}_{2}$, so that $T^{*} B T \in \mathcal{A}$, which shows that $T$ is a semi-normalizer of $\mathcal{B}$ into $\mathcal{A}$. Thus $\mathcal{U}_{\varphi} \subseteq S N(\mathcal{B}, \mathcal{A})$.

Conversely, let $T \in S N(\mathcal{B}, \mathcal{A})$. Define $\varphi \in \mathcal{M}\left(\mathcal{L}_{1}, \mathcal{L}_{2}\right)$ by

$$
\varphi(L)=\overline{[\mathcal{B} T(L)]}, \quad L \in \mathcal{L}_{1} .
$$

It is obvious that $\varphi(L) \in \mathcal{L}_{2}$. It is also easy to see that $\varphi$ is 0 -preserving and joincontinuous. We will show that $T \in \mathcal{U}_{\varphi}$. Let $L \in \mathcal{L}_{1}$. Since $T\left(L \mathcal{H}_{1}\right) \subseteq \mathcal{B} T\left(L \mathcal{H}_{1}\right)$, it is clear that $\varphi(L)^{\perp} T L=0$. On the other hand, for each $B \in \mathcal{B}$ we have $T^{*} B T \in \mathcal{A}$ and so $L^{\perp} T^{*} B T L=0$; hence $\left\langle B T L x, T L^{\perp} y\right\rangle=0$ for each $x, y$. Since the closure of $\left\{B T L x: B \in \mathcal{B}, x \in \mathcal{H}_{1}\right\}$ is $\varphi(L)\left(\mathcal{H}_{2}\right)$, it follows that $\varphi(L)\left(\mathcal{H}_{2}\right) \perp T L^{\perp}\left(\mathcal{H}_{1}\right)$. Thus $\left\langle\varphi(L) z, T L^{\perp} y\right\rangle=0$ for all $y \in \mathcal{H}_{1}, z \in \mathcal{H}_{2}$ and so $\varphi(L) T L^{\perp}=0$. Adding the relation $\varphi(L) T L=T L$, we obtain $\varphi(L) T=T L$ so that $T \in \mathcal{U}_{\varphi}$ as required. $\diamond$

It is obvious that the intersection of several normalizing reflexive subspaces is again a normalizing reflexive subspace. If, for each $\psi \in \mathcal{M}\left(\mathcal{L}_{2}, \mathcal{L}_{1}\right)$, we put

$$
\mathcal{V}_{\psi}=\left\{S \in \mathcal{B}\left(\mathcal{H}_{2}, \mathcal{H}_{1}\right): S M=\psi(M) S \text { for all } M \in \mathcal{L}_{2}\right\},
$$

the above theorem gives us the following

Corollary 5.3. The set $N(\mathcal{B}, \mathcal{A})$ of normalizers of $\mathcal{B}$ into $\mathcal{A}$ is a union of reflexive normalizing subspaces. More precisely,

$$
N(\mathcal{B}, \mathcal{A})=\cup\left\{\mathcal{U}_{\varphi} \cap \mathcal{V}_{\psi}^{*}: \varphi \in \mathcal{M}\left(\mathcal{L}_{1}, \mathcal{L}_{2}\right), \psi \in \mathcal{M}\left(\mathcal{L}_{2}, \mathcal{L}_{1}\right)\right\}
$$

This corollary was proved in [3] for the case of nest algebras.

Recall (Proposition 2.6) that $\mathrm{w}^{*}$-closed normalizing subspaces are generated in norm by their partial isometries. Thus, the previous results have the following consequence:

Corollary 5.4. Any seminormalizer (resp. normalizer) of $\mathcal{B}$ into $\mathcal{A}$ is the normlimit of linear combinations of partial isometries in $S N(\mathcal{B}, \mathcal{A})$ (resp. in $N(\mathcal{B}, \mathcal{A})$ ).

Having shown that the set of normalizers between reflexive algebras is a union of normalizing spaces, we now turn to the converse question: when does a normalizing reflexive space $\mathcal{U}$ consist of semi-normalizers or normalizers of two reflexive algebras $\mathcal{A}$ and $\mathcal{B}$ ? For example, it is easily verified that every operator in $\mathcal{U}$ is a normalizer of the algebra $\operatorname{Alg} \mathcal{S}_{2 \chi}$ into the algebra $\operatorname{Alg} \mathcal{S}_{1 \chi}$ (where $\chi=\operatorname{Map} \mathcal{U}$ ).

We write $\mathcal{A}_{d}=\mathcal{A} \cap \mathcal{A}^{*}$ and $\mathcal{B}_{d}=\mathcal{B} \cap \mathcal{B}^{*}$. If $\mathcal{K}_{1}=\mathcal{U}^{*}\left(\mathcal{H}_{2}\right)=0 \perp \mathcal{H}_{1}$ and $\mathcal{K}_{2}=$ $\mathcal{U}\left(\mathcal{H}_{1}\right)=I_{-} \mathcal{H}_{2}$, we denote by $\mathcal{A}_{o}$ (resp. $\left.\mathcal{B}_{o}\right)$ the compression of $\mathcal{A}$ (resp. $\mathcal{B}$ ) to $\mathcal{K}_{1}$ (resp. $\mathcal{K}_{2}$ ); we write $\mathcal{U}_{o} \subseteq \mathcal{B}\left(\mathcal{K}_{1}, \mathcal{K}_{2}\right)$ for the restriction of $\mathcal{U}$ to $\mathcal{K}_{1}$ and $\chi_{o}$ for the map of $\mathcal{U}_{0}$. 
Lemma 5.5. The following are equivalent:

(1) $\mathcal{U}^{*} \mathcal{U} \subseteq \mathcal{A}$.

(2) For each $L \in$ Lat $\mathcal{A}_{d}, \chi(L) \perp \chi\left(L^{\perp}\right)$.

(3) For each $L \in$ Lat $\mathcal{A}, \chi(L) \perp \chi\left(L^{\perp}\right)$.

(4) With respect to the decomposition $\mathcal{H}_{1}=\mathcal{K}_{1} \oplus \mathcal{K}_{1}^{\perp}$, each $L \in$ Lat $\mathcal{A}$ decomposes as $L=L_{1} \oplus L_{2}$, where $L_{1} \in \mathcal{S}_{1 \chi_{o}}$.

Proof. Since $\mathcal{U}^{*} \mathcal{U} \subseteq \mathcal{A}$ is equivalent to $\mathcal{U}^{*} \mathcal{U} \subseteq \mathcal{A}_{d}$ and hence to Lat $\mathcal{A}_{d} \subseteq$ Lat $\mathcal{U}^{*} \mathcal{U}$ and to Lat $\mathcal{A} \subseteq$ Lat $\mathcal{U}^{*} \mathcal{U}$, the Lemma is an immediate application of Theorem 2.10, since Lat $\mathcal{U}^{*} \mathcal{U}=\left\{L \in \mathcal{P}_{1}: \chi(L) \perp \chi\left(L^{\perp}\right)\right\}=\left\{L_{1} \oplus L_{2}: L_{1} \in \mathcal{S}_{1 \chi_{o}}\right\} . \diamond$

Theorem 5.6. Let $\mathcal{U} \subseteq \mathcal{B}\left(\mathcal{H}_{1}, \mathcal{H}_{2}\right)$ be a $w^{*}$-closed normalizing space. The following are equivalent:

(1) $\mathcal{U} \subseteq S N(\mathcal{B}, \mathcal{A})$

(2) (i) $\chi(L) \perp \chi\left(L^{\perp}\right)$ for each $L \in$ Lat $\mathcal{A}$ and (ii) $\chi_{o}$ (Lat $\left.\mathcal{A}_{o}\right) \subseteq$ Lat $\mathcal{B}_{o}$.

(3) (i) $\chi(L) \perp \chi\left(L^{\perp}\right)$ for each $L \in$ Lat $\mathcal{A}$ and (ii) for each $L \in$ Lat $\mathcal{A}$, there exists a projection $Q$ such that $\overline{[\mathcal{B} \chi(L)]}=\left.\chi(L)\right|_{\mathcal{K}_{1}} \oplus Q$.

Proof. (1) $\Leftrightarrow(\mathbf{3})$ Suppose (1) holds. Then $\mathcal{U}^{*} \mathcal{U} \subseteq \mathcal{A}$, and (i) holds by the Lemma. For all $T, S \in \mathcal{U}, B \in \mathcal{B}$ and $L \in$ Lat $\mathcal{A}$ we have

$$
\left\langle B S L x, T L^{\perp} y\right\rangle=0 \quad\left(x, y \in \mathcal{H}_{1}\right) .
$$

Thus $\overline{[\mathcal{B} \chi(L)]} \leq \chi\left(L^{\perp}\right)^{\perp}=\left(\chi(L)^{\perp} \wedge \chi(I)\right)^{\perp}=\chi(L) \vee \chi(I)^{\perp}$. Writing $L=L_{1} \oplus L_{2}$ where $L_{1} \in \mathcal{S}_{1 \chi_{o}}$ we have $\chi(L)=\chi_{o}\left(L_{1}\right) \oplus 0$. Since $\chi(L) \leq \overline{[\mathcal{B} \chi(L)]}$ we obtain

$$
\chi_{o}\left(L_{1}\right) \oplus 0 \leq \overline{[\mathcal{B} \chi(L)]} \leq \chi_{o}\left(L_{1}\right) \oplus I
$$

which shows that $\overline{[\mathcal{B} \chi(L)]}=\chi_{o}\left(L_{1}\right) \oplus Q$, for some projection $Q$, as required.

These steps can clearly be reversed.

(1) $\Rightarrow$ (2) Again (i) holds by the Lemma. For (ii), note first that $\mathcal{U}_{o} \subseteq S N\left(\mathcal{B}_{o}, \mathcal{A}_{o}\right)$. Thus for each $L \in$ Lat $\mathcal{A}_{o}, S, T \in \mathcal{U}_{o}$ and $B \in \mathcal{B}_{o}$ we have $L^{\perp} T^{*} B S L=0$. As in the proof of $(1) \Rightarrow(3)$, we conclude that $\chi_{o}(L) \leq \overline{\left[\mathcal{B}_{o} \chi_{o}(L)\right]} \leq \chi_{o}\left(L^{\perp}\right)^{\perp}=\chi_{o}(L)$ since $\chi_{o}(I)=I$. Thus $\chi_{o}(L)=\overline{\left[\mathcal{B}_{o} \chi_{o}(L)\right]} \in$ Lat $\mathcal{B}_{o}$.

(2) $\Rightarrow(\mathbf{1})$ Given $B \in \mathcal{B}$ and $T \in \mathcal{U}$, we will prove that $T^{*} B T \in \mathcal{A}$, equivalently that $L^{\perp} T^{*} B T L=0$ for each $L \in$ Lat $\mathcal{A}$. Since $\mathcal{U}^{*} \mathcal{U} \subseteq \mathcal{A}$, we have $L=L_{1} \oplus L_{2}$ where $L_{1} \in \mathcal{S}_{1 \chi_{o}}$ by the Lemma. It is easily seen that $L_{1} \in$ Lat $\mathcal{A}_{o}$.

Since $T \in \mathcal{U}$, we have $T\left(L^{\perp} \mathcal{H}_{1}\right) \subseteq \chi\left(L^{\perp}\right) \mathcal{H}_{2} \subseteq \chi(L)^{\perp} \mathcal{H}_{2}$ since $\chi\left(L^{\perp}\right) \perp \chi(L)$ by the Lemma. Thus $\chi(L) T L^{\perp}=0$. Since also $\chi(L)^{\perp} T L=0$, it follows that $T L=\chi(L) T$ and $T L^{\perp}=\chi(L)^{\perp} \chi(I) T$ since the range of $T$ is contained in $\chi(I)$. Thus $L^{\perp} T^{*} B T L=T^{*} \chi(L)^{\perp} \chi(I) B \chi(I) \chi(L) T$. But $\left.\chi(I) B\right|_{\mathcal{K}_{2}} \in \mathcal{B}_{O}$ and since $\left.\chi(L)\right|_{\mathcal{K}_{2}}=$ $\chi_{o}\left(L_{1}\right) \in$ Lat $\mathcal{B}_{o}$ by assumption we have $(\chi(I) B \chi(I)) \chi(L)=\chi(L)(\chi(I) B \chi(I)) \chi(L)$ and so $L^{\perp} T^{*} B T L=0$. This shows that $T^{*} B T \in \mathcal{A}$ and concludes the proof. $\diamond$

Corollary 5.7. Let $\mathcal{U} \subseteq \mathcal{B}\left(\mathcal{H}_{1}, \mathcal{H}_{2}\right)$ be an essential normalizing ultraweakly closed subspace and $\chi=\operatorname{Map} \mathcal{U}$. Then $\mathcal{U} \subseteq S N(\mathcal{B}, \mathcal{A})$ if and only if

(i) $\chi(L) \perp \chi\left(L^{\perp}\right)$ for each $L \in$ Lat $\mathcal{A}$ and

(ii) $\chi($ Lat $\mathcal{A}) \subseteq$ Lat $\mathcal{B}$.

Proof. Immediate from Theorem 5.6. $\diamond$ 
Corollary 5.8. Let $\mathcal{U} \subseteq \mathcal{B}\left(\mathcal{H}_{1}, \mathcal{H}_{2}\right)$ be a normalizing ultraweakly closed subspace and let $\chi=\operatorname{Map} \mathcal{U}$ and $\chi^{*}=\operatorname{Map} \mathcal{U}^{*}$. Then $\mathcal{U} \subseteq N(\mathcal{B}, \mathcal{A})$ if and only if

(i) $\chi(L) \perp \chi\left(L^{\perp}\right)$ for each $L \in$ Lat $\mathcal{A}$ while $\chi^{*}(M) \perp \chi^{*}\left(M^{\perp}\right)$ for each $M \in$ Lat $\mathcal{B}$ and

(ii) $\chi_{o}\left(\right.$ Lat $\left.\mathcal{A}_{o}\right)=$ Lat $\mathcal{B}_{o}$.

Proof. If $\mathcal{U} \subseteq N(\mathcal{B}, \mathcal{A})$, then $\chi_{o}\left(\right.$ Lat $\left.\mathcal{A}_{o}\right) \subseteq$ Lat $\mathcal{B}_{o}$ and $\chi_{o}^{*}\left(\right.$ Lat $\left.\mathcal{B}_{o}\right) \subseteq$ Lat $\mathcal{A}_{o}$ by Theorem 5.6. But $\mathcal{U}_{o}^{*} \mathcal{U}_{o} \subseteq \mathcal{A}_{o}$ and hence Lat $\mathcal{A}_{o} \subseteq \operatorname{Lat}\left(\mathcal{U}_{o}^{*} \mathcal{U}_{o}\right)=\mathcal{S}_{1 \chi_{o}}$ (Theorem 2.10). It follows that $\chi_{o}$ is one-to-one on Lat $\mathcal{A}_{o}$ into Lat $\mathcal{B}_{o}$. Similarly, Lat $\mathcal{B}_{o} \subseteq$ $\operatorname{Lat}\left(\mathcal{U}_{o} \mathcal{U}_{o}^{*}\right)=\mathcal{S}_{2 \chi_{o}}$ and hence the inverse of $\chi_{o}$, namely $\chi_{o}^{*}$, maps Lat $\mathcal{B}_{o} 1-1$ into Lat $\mathcal{A}_{o}$.

Suppose conversely that (i) and (ii) hold. By Theorem 5.6, $\mathcal{U} \subseteq S N(\mathcal{B}, \mathcal{A})$. As above, (i) gives Lat $\mathcal{A}_{o} \subseteq \mathcal{S}_{1 \chi_{o}}$ and hence $\left.\chi_{o}^{*} \chi_{o}\right|_{\text {Lat }} \mathcal{A}_{o}=\left.i d\right|_{\text {Lat }} \mathcal{A}_{o}$ from Theorem 2.10. Therefore $\chi^{*}\left(\right.$ Lat $\left.\mathcal{B}_{o}\right) \subseteq$ Lat $\mathcal{A}_{o}$ and so $\mathcal{U}^{*} \subseteq S N(\mathcal{A}, \mathcal{B})$ again from Theorem 5.6. $\diamond$

Corollary 5.9. Let $\mathcal{U} \subseteq \mathcal{B}\left(\mathcal{H}_{1}, \mathcal{H}_{2}\right)$ be an essential normalizing ultraweakly closed subspace and let $\chi=\operatorname{Map} \mathcal{U}$. Then $\mathcal{U} \subseteq N(\mathcal{B}, \mathcal{A})$ if and only if

(i) $\chi(L) \perp \chi\left(L^{\perp}\right)$ for each $L \in$ Lat $\mathcal{A}$ and

(ii) $\chi($ Lat $\mathcal{A})=\operatorname{Lat} \mathcal{B}$.

Proof. From Corollary 5.8 it is sufficient to show that $(i)$ and (ii) imply that $\chi^{*}(M) \perp \chi^{*}\left(M^{\perp}\right)$ for each $M \in$ Lat $\mathcal{B}_{d}$. But this is immediate from Lemma 5.5 (applied to $\left.\mathcal{U}^{*}\right)$ since from $($ ii $)$ we have Lat $\mathcal{B}=\chi($ Lat $\mathcal{A}) \subseteq \mathcal{S}_{2}=\operatorname{Lat}\left(\mathcal{U} \mathcal{U}^{*}\right)$ and so $\mathcal{U U}^{*} \subseteq \mathcal{B} . \diamond$

Note that Theorem 5.2 yields, for every $T \in S N(\mathcal{B}, \mathcal{A})$, a certain normalizing subspace $\mathcal{U}_{\varphi}$, such that $T \in \mathcal{U}_{\varphi} \subseteq S N(\mathcal{B}, \mathcal{A})$. We show that this property extends from single operators to linear spaces consisting of seminormalizers. Recall that $\mathcal{U}_{\varphi}$ is a $\mathcal{B}_{d}, \mathcal{A}_{d}$-bimodule, where $\mathcal{A}_{d}$ and $\mathcal{B}_{d}$ are the diagonals of $\mathcal{A}$ and $\mathcal{B}$. It may be interesting to note that all right $\mathcal{A}_{d}$-modules $\mathcal{U}$ which consist of semi-normalizers of $\mathcal{B}$ into $\mathcal{A}$ automatically satisfy a rather strong condition.

Proposition 5.10. (1) Any linear space $\mathcal{U} \subseteq S N(\mathcal{B}, \mathcal{A})$ (resp. $\mathcal{U} \subseteq N(\mathcal{B}, \mathcal{A})$ ) is contained in an ultraweakly closed normalizing space $\mathcal{U}_{A} \subseteq S N(\mathcal{B}, \mathcal{A})$ (resp. $\mathcal{U}_{A} \subseteq$ $N(\mathcal{B}, \mathcal{A})$ ) which is a right $\mathcal{A}_{d}$-module.

(2) If $\mathcal{U} \subseteq S N(\mathcal{B}, \mathcal{A})$ is a right $\mathcal{A}_{d}$-module, then $\mathcal{U}$ is automatically normalizing. Moreover, $0_{+}=\operatorname{ker} \mathcal{U}$ is in the centre of $\mathcal{A}_{d}$ and the restriction $\mathcal{A}_{\text {od }}$ of $\mathcal{A}_{d}$ to $\mathcal{K}_{1}=$ $0_{+} \mathcal{H}_{1}$ is the bicommutant of $\left.\left(\mathcal{U}^{*} \mathcal{U}\right)\right|_{\mathcal{K}_{1}}$. It follows that $\mathcal{S}_{1 \chi}=\left\{L \oplus I: L \in\right.$ Lat $\left.\mathcal{A}_{o d}\right\}$.

Proof. (1) Suppose $\mathcal{U} \subseteq S N(\mathcal{B}, \mathcal{A})$ is a linear space. Define $\mathcal{U}_{a}=\left[\mathcal{U} \mathcal{A}_{d}\right]$ and $\mathcal{U}_{A}=$ ${\overline{\left[\mathcal{U} \mathcal{A}_{d}\right]}}^{u w}$. If $T_{1}, T_{2} \in \mathcal{U}$ and $A_{1}, A_{2} \in \mathcal{A}_{d}$, then for each $B \in \mathcal{B}$ the fact that $T_{i}^{*} B T_{j} \in \mathcal{A}$ and $A_{i}, A_{i}^{*} \in \mathcal{A}$ yields $\left(T_{1} A_{1}+T_{2} A_{2}\right)^{*} B\left(T_{1} A_{1}+T_{2} A_{2}\right) \in \mathcal{A}$. Thus $\mathcal{U}_{a} \subseteq S N(\mathcal{B}, \mathcal{A})$.

Now $\mathcal{U}_{a} \mathcal{A}_{d} \subseteq \mathcal{U}_{a}$ by construction and $\mathcal{U}_{a}^{*} \mathcal{U}_{a} \subseteq \mathcal{A}$ since $\mathcal{U}_{a} \subseteq S N(\mathcal{B}, \mathcal{A})$. Therefore $\mathcal{U}_{a}$ is normalizing (Remark 2.2).

It follows from Theorem 2.4 that $\mathcal{U}_{A}$ is a reflexive normalizing space. Also, since $\mathcal{U}_{A}$ is the strong operator closure of $\mathcal{U}_{a}$ and $S N(\mathcal{B}, \mathcal{A})$ is strongly closed, $\mathcal{U}_{A} \subseteq S N(\mathcal{B}, \mathcal{A})$. That the strong closure of a right $\mathcal{A}_{d}$-module is a right $\mathcal{A}_{d}$-module is obvious.

The case $\mathcal{U} \subseteq N(\mathcal{B}, \mathcal{A})$ is similar.

(2) That $\mathcal{U}$ is normalizing follows from Remark 2.2 as above. Let $\chi=\operatorname{Map} \mathcal{U}$. Since $\mathcal{U} \mathcal{A}_{d} \subseteq \mathcal{U}$ we have $\mathcal{S}_{1 \chi} \subseteq$ Lat $\mathcal{A}_{d}$ (Lemma 1.1). On the other hand since 
$\mathcal{U} \subseteq S N(\mathcal{B}, \mathcal{A})$ we have $\mathcal{U}^{*} \mathcal{U} \subseteq \mathcal{A}_{d}$. Thus $0_{+}$commutes with $\mathcal{A}_{d}$ and also $0_{+} \in$ $\left(\mathcal{U}^{*} \mathcal{U}\right)^{\prime \prime} \subseteq \mathcal{A}_{d}$, so $0_{+}$is in the centre of $\mathcal{A}_{d}$. Hence $\mathcal{A}_{d}$ can be written $\mathcal{A}_{o d} \oplus \mathcal{A}_{1 d}$ with respect to the decomposition $\mathcal{H}_{1}=\mathcal{K}_{1} \oplus \mathcal{K}_{1}^{\perp}$. Using Theorem 2.10, we have

$$
\left(\mathcal{U}^{*} \mathcal{U}\right)^{\prime \prime}=\left(\begin{array}{cc}
\left(\mathcal{S}_{1 \chi} \mid \mathcal{K}_{1}\right)^{\prime} & 0 \\
0 & \mathbb{C} I
\end{array}\right) \subseteq \mathcal{A}_{d} \subseteq \mathcal{S}_{1 \chi}^{\prime}=\left(\begin{array}{cc}
\left(\mathcal{S}_{1 \chi} \mid \mathcal{K}_{1}\right)^{\prime} & 0 \\
0 & \mathcal{B}\left(\mathcal{K}_{1}^{\perp}\right)
\end{array}\right)
$$

This shows that $\mathcal{A}_{\text {od }}$ equals $\left(\mathcal{S}_{1 \chi} \mid \mathcal{K}_{1}\right)^{\prime}=\left(\left.\mathcal{U}^{*} \mathcal{U}\right|_{\mathcal{K}_{1}}\right)^{\prime \prime}$ and Lat $\mathcal{A}_{\text {od }}$ equals $\mathcal{S}_{1 \chi} \mid \mathcal{K}_{1}=$ $\operatorname{Lat}\left(\left.\mathcal{U}^{*} \mathcal{U}\right|_{\mathcal{K}_{1}}\right)$. Thus $\mathcal{S}_{1 \chi}=\left\{L \oplus I: L \in \operatorname{Lat}\left(\left.\mathcal{U}^{*} \mathcal{U}\right|_{\mathcal{K}_{1}}\right)\right\}=\left\{L \oplus I: L \in \operatorname{Lat} \mathcal{A}_{o d}\right\}$.

It is clear that every linear space $\mathcal{U} \subseteq S N(\mathcal{B}, \mathcal{A})$ is also contained in a $\mathrm{w}^{*}$-closed normalizing space which is a $\left(\mathcal{B}_{d}, \mathcal{A}_{d}\right)$-bimodule (just consider the $\mathrm{w}^{*}$-closure of $\left.\left[\mathcal{B}_{d} \mathcal{U A}_{d}\right]\right)$. This bimodule is not necessarily maximal with respect to being a linear space of seminormalizers.

For an example, consider the nest algebra $\mathcal{A}$ of all upper triangular $2 \times 2$ matrices and let $\mathcal{U}$ be the space of all strictly lower triangular matrices. One easily checks that $\mathcal{U} \subseteq S N(\mathcal{A}, \mathcal{A})$ and that $\mathcal{U}$ is a bimodule over the diagonal algebra (which is a masa in this case). However, it is readily verified that the linear span of the matrix unit $E_{11}$ and $\mathcal{U}$ is also contained in $S N(\mathcal{A}, \mathcal{A})$.

Remark 5.11. Let $\mathcal{U} \subseteq S N(\mathcal{B}, \mathcal{A})$ be an essential $w^{*}$-closed normalizing space, which is a right $\mathcal{A}_{d}$-module. Then $\mathcal{U}$ is maximal with respect to being a linear space in $S N(\mathcal{B}, \mathcal{A})$.

Proof. Let $\mathcal{V} \subseteq S N(\mathcal{B}, \mathcal{A})$ be a linear space containing $\mathcal{U}$ and let $T \in \mathcal{V}$. For each $S \in$ $\mathcal{U}$, we have $S^{*} T \in \mathcal{V}^{*} \mathcal{V} \subseteq \mathcal{A}_{d}$. Since $\mathcal{U} \mathcal{A}_{d} \subseteq \mathcal{U}$, if $\chi=$ Map $\mathcal{U}$ we have $\mathcal{S}_{1 \chi} \subseteq$ Lat $\mathcal{A}_{d}$ (Lemma 1.1). Thus $L^{\perp} S^{*} T L=0$ for each $L \in \mathcal{S}_{1 \chi}$ and hence $\left\langle T L x, S L^{\perp} y\right\rangle=0$ for all $x, y$. But the closure of $\left\{S L^{\perp} y: S \in \mathcal{U}, y \in \mathcal{H}_{1}\right\}$ is $\chi\left(L^{\perp}\right) \mathcal{H}_{2}$ which equals $\chi(L)^{\perp} \mathcal{H}_{2}$ since $\chi$ is essential; therefore $T L\left(\mathcal{H}_{1}\right) \perp \chi(L)^{\perp} \mathcal{H}_{2}$. We have shown that $\chi(L)^{\perp} T L=0$ for all $L \in \mathcal{S}_{1 \chi}$ and so $T \in \mathcal{U} . \diamond$

Suppose now that $\mathcal{A}$ and $\mathcal{B}$ are CSL algebras, that is, the respective invariant subspace lattices are commutative. In this case Proposition 3.4 and Theorem 4.1 immediately yield the next corollary. The last statement was proved by Coates [3] for the case of nest algebras.

Corollary 5.12. If $\mathcal{A}$ and $\mathcal{B}$ are $C S L$ algebras, the $\operatorname{set} S N(\mathcal{B}, \mathcal{A})(N(\mathcal{B}, \mathcal{A}))$ of seminormalizers (normalizers) of $\mathcal{B}$ into $\mathcal{A}$ is a union of synthetic normalizing masabimodules. Each compact operator $K$ in $S N(\mathcal{B}, \mathcal{A})(N(\mathcal{B}, \mathcal{A})$ ) can be approximated in norm by sums of rank one operators in $S N(\mathcal{B}, \mathcal{A})(N(\mathcal{B}, \mathcal{A}))$. Moreover, if $K \in$ $\mathcal{C}_{p}$, then it can be approximated in the $\mathcal{C}_{p}$-norm by sums of rank one operators in $S N(\mathcal{B}, \mathcal{A})(N(\mathcal{B}, \mathcal{A}))$. Finally, if $K$ has finite rank, say $n$, it can be written as a sum of $n$ rank one operators in $S N(\mathcal{B}, \mathcal{A})(N(\mathcal{B}, \mathcal{A}))$.

We would like to conclude this paper with a discussion of the behaviour of the set of semi-normalizers (normalizers) with respect to addition. It is clear that, if $T$ and $S$ are semi-normalizers (normalizers) of an algebra into another, the sum $T+S$ is not necessarily a semi-normalizer (normalizer). Proposition 5.14 below gives a necessary and sufficient condition for this to happen, when the algebras in question are CSL algebras. First we prove a statement, concerning arbitrary masa-bimodules. 
Lemma 5.13. Let $\Gamma: \mathcal{B}\left(\mathcal{H}_{1}, \mathcal{H}_{2}\right) \longrightarrow\{0,1\}$ be such that, if $\Gamma(T)=1$ for some operator $T$, then there is a reflexive masa-bimodule $\mathcal{U}$, containing $T$, such that $\Gamma(S)=$ 1 for all $S \in \mathcal{U}$. Suppose that $T_{1}$ and $T_{2}$ are operators, such that $\Gamma\left(\lambda_{1} T_{1}+\lambda_{2} T_{2}\right)=1$ for all real numbers $\lambda_{1}$ and $\lambda_{2}$. Then there exists a reflexive masa-bimodule $\mathcal{U}$, containing $T_{1}$ and $T_{2}$, such that $\Gamma(T)=1$ for all $T \in \mathcal{U}$.

Proof. Represent $\mathcal{H}_{1}$ as $L^{2}(X, \mu)$ and $\mathcal{H}_{2}$ as $L^{2}(Y, \nu)$, where $(X, \mu)$ and $(Y, \nu)$ are compact metric spaces with regular Borel measures, and let $\mathcal{D}_{1}$ and $\mathcal{D}_{2}$ be the respective multiplication algebras. By the support $\operatorname{supp} T$ of an operator $T$ we will mean the (closed) support of the reflexive $\mathcal{D}_{2}, \mathcal{D}_{1}$-bimodule generated by $T$. Let $\operatorname{supp} T_{1}=\kappa_{1}, \operatorname{supp} T_{2}=\kappa_{2}$ and $\kappa=\kappa_{1} \cup \kappa_{2}$. The sets $\kappa_{1}, \kappa_{2}$ and $\kappa$ are compact subsets of $X \times Y$. It suffices to find a real number $\lambda$ such that the operator $T_{1}+\lambda T_{2}$ has support $\kappa$. Indeed, in this case, there exists a reflexive $\mathcal{D}_{2}, \mathcal{D}_{1}$-bimodule $\mathcal{U}$ containing $T_{1}+\lambda T_{2}$ such that $\Gamma(T)=1$ for all $T \in \mathcal{U}$. Since $\kappa \subseteq \operatorname{supp} \mathcal{U}$ it follows from [5], Theorem 4.6 that $T_{1}, T_{2} \in \mathcal{U}$.

Put $\kappa_{\lambda}=\operatorname{supp}\left(T_{1}+\lambda T_{2}\right), v_{\lambda}=\kappa_{\lambda}^{c}$. It is clear that $v_{\lambda}$ is an open subset of $X \times Y$. We claim that, if $\lambda \neq \lambda^{\prime}$ are nonzero, then $v_{\lambda} \cap v_{\lambda^{\prime}} \subseteq \kappa^{c}$. Indeed whenever $\alpha$ and $\beta$ are open and such that $\alpha \times \beta \subseteq v_{\lambda} \cap v_{\lambda^{\prime}}$, then $F(\beta)\left(T_{1}+\lambda T_{2}\right) E(\alpha)=0$ and also $F(\beta)\left(T_{1}+\lambda^{\prime} T_{2}\right) E(\alpha)=0$. Thus $\left(\lambda-\lambda^{\prime}\right) F(\beta) T_{2} E(\alpha)=0$ and hence $F(\beta) T_{2} E(\alpha)=0$, so that $\alpha \times \beta \cap \kappa_{2}=\emptyset$. Repeating the argument to the operators $\frac{1}{\lambda} T_{1}+T_{2}$ and $\frac{1}{\lambda^{\prime}} T_{1}+T_{2}$ gives $\alpha \times \beta \cap \kappa_{1}=\emptyset$. It follows that $v_{\lambda} \cap v_{\lambda^{\prime}} \cap \kappa_{2}=\emptyset$ and $v_{\lambda} \cap v_{\lambda^{\prime}} \cap \kappa_{1}=\emptyset$, so that $v_{\lambda} \cap v_{\lambda^{\prime}} \subseteq \kappa^{c}$.

Thus the union $\cup\left\{v_{\lambda} \cap \kappa: \lambda \in \mathbb{R} \backslash\{0\}\right\}$ is an uncountable union of (relatively) open disjoint subsets of $\kappa$. Since $\kappa$ is a compact metric space, no more than countably many of them can be nonempty. Thus there exists $\lambda \in \mathbb{R} \backslash\{0\}$ so that $v_{\lambda} \cap \kappa=\emptyset$ or $\kappa \subseteq \kappa_{\lambda}$. But $\kappa_{\lambda} \subseteq \kappa$. Indeed if $(\alpha \times \beta) \cap \kappa=\emptyset$ with $\alpha$ and $\beta$ open, then $F(\beta) T_{i} E(\alpha)=0$ for $i=1,2$ so $F(\beta)\left(T_{1}+\lambda T_{2}\right) E(\alpha)=0$ which means that $(\alpha \times \beta) \cap \kappa_{\lambda}=\emptyset$. $\diamond$

Proposition 5.14. Let $\mathcal{A}$ and $\mathcal{B}$ be $C S L$ algebras and $T, S \in S N(\mathcal{B}, \mathcal{A})$ (resp. $T, S \in$ $N(\mathcal{B}, \mathcal{A}))$. Then $T+S \in S N(\mathcal{B}, \mathcal{A})(T+S \in N(\mathcal{B}, \mathcal{A}))$ if and only if there is a reflexive normalizing masa-bimodule $\mathcal{U} \subseteq S N(\mathcal{B}, \mathcal{A}) \quad \mathcal{U} \subseteq N(\mathcal{B}, \mathcal{A})$ ) such that $T, S \in \mathcal{U}$.

Proof. We will consider the case of semi-normalizers, the case of normalizers is similar. It is clear that if there exists a reflexive normalizing masa-bimodule $\mathcal{U} \subseteq$ $S N(\mathcal{B}, \mathcal{A})$ such that $T, S \in \mathcal{U}$, then $T+S$ is a semi-normalizer. Conversely, suppose that $T, S$ and $T+S$ are semi-normalizers of $\mathcal{B}$ into $\mathcal{A}$. An elementary computation shows that then $T^{*} B S+S^{*} B T \in \mathcal{A}$ for each $B \in \mathcal{B}$. But then it is easy to see that $\lambda_{1} T+\lambda_{2} S$ is a semi-normalizer for all real numbers $\lambda_{1}$ and $\lambda_{2}$. From Theorem 5.2 we have that, if $T \in S N(\mathcal{B}, \mathcal{A})$, then there exists a reflexive space $\mathcal{U} \subseteq S N(\mathcal{B}, \mathcal{A})$, containing $T$. The space $\mathcal{U}$ is a masa bimodule, since the semi-lattices of its map are commutative. Thus Lemma 5.13 applies with the property of being a semi-normalizer of $\mathcal{B}$ into $\mathcal{A}$ in the place of $\Gamma . \diamond$

Corollary 5.15. Let $\mathcal{A}$ and $\mathcal{B}$ be $C S L$ algebras and $T, S$ be semi-normalizers (normalizers) of $\mathcal{B}$ into $\mathcal{A}$, such that $T+S$ is also a semi-normalizer (normalizer). Then $B_{1} T A_{1}+B_{2} S A_{2}$ is a semi-normalizer (normalizer) for every $B_{1}, B_{2} \in \mathcal{B} \cap \mathcal{B}^{*}$ and $A_{1}, A_{2} \in \mathcal{A} \cap \mathcal{A}^{*}$. 
Addendum After this paper was completed, we were informed that what we call "normalizing spaces of operators" have been studied, from a different viewpoint, by other authors under the names "ternary rings of operators" or "triple systems". See for example M. Neal and B. Russo, Contractive Projections and Operator Spaces (preprint, arXiv: math.OA/0201187) and its bibliography. We thank J. Arazy, D.P. Blecher and B. Russo for bringing the relevant literature to our attention. We have chosen to retain the name "normalizing spaces" to emphasize the relation with normalizers of operator algebras, which is one of the main points of our work.

We have also found out that our Proposition 2.6 is essentially contained in section 3 of the paper of L.A. Harris, 'A generalization of $\mathrm{C}^{*}$-algebras', Proc. London Math. Soc. (3) 42 (1981) 331-361.

\section{REFERENCES}

[1] W. B. Arveson, Operator algebras and invariant subspaces, Ann. Math. (2) 100 (1974), 433-532.

[2] G. Birkhoff, Lattice Theory, 3rd edition, Colloquium Publications 25 (American Mathematical Society, Providence, 1979).

[3] K.J. Coates, Normalizers of nest algebras, Proc. Amer. Math. Soc. 126 (1998), 159-165; Errata, ibid, 2511-2512.

[4] K.R. Davidson, Nest Algebras, Pitman Research Notes in Mathematics, 191, Longman (1988).

[5] J.A. Erdos, A. Katavolos, V.S. Shulman, Rank one subspaces of Bimodules over Maximal Abelian Selfadjoint Algebras, J. Funct. Anal. 157 No.2 (1998), 554-587.

[6] J.A. Erdos, Reflexivity for subspace maps and linear spaces of operators, Proc. London Math. Soc. (3) 52 (1984), 582-600.

[7] J. Feldman and C.C. Moore, Ergodic equivalence relations, cohomology and von Neumann algebras I and II, Trans. Amer. Math. Soc. 234 No. 2 (1977), 289-324, 325-359.

[8] P.R. Halmos, Reflexive lattices of subspaces, J. London Math. Soc. (2) 4 (1971), 257-263.

[9] A. Hopenwasser, C. Laurie and R. Moore, Reflexive algebras with completely distributive subspace lattice, J. Oper. Th. 11 (1984), 91-108.

[10] R. Kadison and I. Singer, Triangular operator algebras, Amer. J. Math. 82 (1960), 227-259.

[11] W.E. Longstaff, Operators of rank one in reflexive algebras, Canad. J. Math. 28 (1976), 19-23.

[12] A.I. Loginov and V.S. Shul'man, Hereditary and intermediate reflexivity of $\mathrm{W}^{*}$-algebras, Izv. Akad. Nauk. SSSR, 39 (1975), 1260-1273; Math. USSR-Izv. 9 (1975), 1189-1201.

[13] H. Radjavi and P. Rosenthal, Invariant Subpaces, Springer-Verlag, N.Y. (1973).

[14] M.A. Rieffel, Morita equivalence for $\mathrm{C}^{*}$-algebras and $\mathrm{W}^{*}$-algebras, J. Pure and Applied Algebra 5 (1974), 51-96.

[15] R. Sikorski, On the inducing of homomorphisms by mappings, Fund. Math. 36 (1949), 7-22.

[16] I. Todorov, Modules over reflexive algebras, Doctoral Thesis, University of Athens (in Greek) (1999).

Department of Mathematics, University of Athens, 15784 Athens, Greece

E-mail address: akatavol@eudoxos.math.uoa.gr

current address (I.G. Todorov) Department of Pure Mathematics, Queen's UniverSity Belfast, Belfast BT7 1NN Northern Ireland, United Kingdom

E-mail address: i.todorov@qub.ac.uk 